Marsquakes

\title{
On the evolution of thermally stratified layers at the top of Earth's core
}

\author{
Sam Greenwood *, Christopher J. Davies, Jon E. Mound \\ School of Earth and Environment, University of Leeds, Leeds LS2 9JT, UK
}

\section{A R T I C L E I N F O}

\section{Keywords:}

Geodynamo

Outer core

Thermal history

Inner core age

\begin{abstract}
A B S T R A C T
Stable stratification at the top of the Earth's outer core has been suggested based upon seismic and geomagnetic observations, however, the origin of the layer is still unknown. In this paper we focus on a thermal origin for the layer and conduct a systematic study on the thermal evolution of the core. We develop a new numerical code to model the growth of thermally stable layers beneath the CMB, integrated into a thermodynamic model for the long term evolution of the core. We conduct a systematic study on plausible thermal histories using a range of core properties and, combining thickness and stratification strength constraints, investigate the limits upon the present day structure of the thermal layer. We find that whilst there are a number of scenarios for the history of the CMB heat flow, $Q_{c}$, that give rise to thermal stratification, many of them are inconsistent with previously published exponential trends in $Q_{\mathrm{c}}$ from mantle evolution models. Layers formed due to an exponentially decaying $Q_{\mathrm{c}}$ are limited to $250-400 \mathrm{~km}$ thick and have maximum present-day Brunt-Väisälä periods, $T_{\mathrm{BV}}=8-$ $24 \mathrm{hrs}$. When entrainment of the lowermost region of the layer is included in our model, the upper limit of the layer size is reduced and can fully inhibit the growth of any layer if our non-dimensional measure of entrainment, $E>0.2$. The period $T_{\mathrm{BV}}$ is insensitive to the evolution and so our estimates remain distinct from estimates arising from a chemical origin. Therefore, $T_{\mathrm{BV}}$ should be able to discern between thermal and chemical mechanisms as improved seismic constraints are obtained.
\end{abstract}

\section{Introduction}

The Earth's large scale magnetic field is generated within the liquid iron outer core by the geodynamo process, which converts the mechanical energy of fluid motion into magnetic energy. Spatial and temporal variations of the field observed at Earth's surface reflect processes at the top of the core and so establishing the structure and dynamics of this region is of particular importance. Much debate has focused on the presence of stable stratification beneath the core-mantle boundary (CMB). A range of seismic studies (Lay and Young, 1990; Garnero et al., 1993; Helffrich and Kaneshima, 2010; Kaneshima, 2017), but not all (Alexandrakis and Eaton, 2010), find significant P-wave velocity reductions relative to the Preliminary Reference Earth Model (PREM, Dziewonski and Anderson, 1981) ranging up to $400 \mathrm{~km}$ deep into the core. This has been interpreted as a layer of anomalously light fluid (Helffrich and Kaneshima, 2013) that is resistant to the convective motion beneath it, although this interpretation has been recently challenged (Irving et al., 2018). The existence of a stratified layer has important implications for interpreting geomagnetic observations because stable regions filter the signal from the deeper core
(Christensen, 2006) and support unique classes of wave motions such as MAC waves, which have been invoked to explain certain periodic variations in the observed magnetic field and length of day (Buffett et al., 2016).

A number of key properties of the stable layer are uncertain such as its size, age, and thermal and chemical structure, which all depend upon the underlying mechanism generating the stratification. A systematic study of the time evolution of the core in which stable stratification arises is required in order to anticipate these key properties given plausible scenarios. Future constraints from observations on the layer size and Brunt-Väisälä frequencies may then be related to these models to distinguish between different origins for the layer, to infer the structure and dynamics of the upper region of the core, and to relate to paleomagnetic observations.

Several mechanisms have been proposed to explain the origin of a stable layer below the CMB. Chemical stratification may be caused by the barodiffusion of light element towards the CMB (Fearn and Loper, 1981; Gubbins and Davies, 2013), by the accumulation of blobs of chemically distinct material at the CMB (Moffatt and Loper, 1994; Bouffard et al., 2019), by transfer of lighter oxides from the mantle

\footnotetext{
* Corresponding author.

E-mail address: s.greenwoodeleeds.ac.uk (S. Greenwood).
} 
(Buffett and Seagle, 2010; Davies et al., 2018, 2020) or by incomplete mixing during core formation (Landeau et al., 2016). Alternatively, the layer may be thermally stratified if the heat flow at the CMB, $Q_{c}$, falls below the heat that is conducted down the adiabat, $Q_{\mathrm{a}}$ (Gubbins et al., 1982; Labrosse et al., 1997; Lister and Buffett, 1998). Although a chemical origin is typically favoured over a thermal origin to explain the magnitude of the observed seismic velocity anomaly, theoretical calculations have shown an increases in P-wave velocity with increased light element (Buffett and Seagle, 2010; Brodholt and Badro, 2017). Instead, specific enrichments and depletions of multiple light elements are needed to explain the velocity decrease (Brodholt and Badro, 2017). Furthermore, inferred MAC waves favour weak stratification, consistent with thermal stratification (Buffett et al., 2016). For this paper we will therefore focus on a thermal origin for the stable layer.

The present CMB heat flow is estimated to lie in the range $Q_{c}=5-$ 17 TW (Lay et al., 2009; Nimmo, 2015). The heat flow down an adiabatic temperature gradient $Q_{\mathrm{a}}$ depends on the thermal conductivity $k$ and temperature gradient at the top of the core. Assuming an adiabatic temperature gradient of $\sim 1 \mathrm{~K} \mathrm{~km}^{-1}$ (Davies et al., 2015) and $k$ values in the range $40-100 \mathrm{~W} \mathrm{~m}^{-1} \mathrm{~K}^{-1}$ (de Koker et al., 2012; Pozzo et al., 2013; Gomi et al., 2013; Konôpková et al., 2016) gives $Q_{a} \sim 4-16$ TW and so both strong stabilising and destabilising conditions are consistent with the available information. Gubbins et al. (2015) used these values and theoretical conduction profiles describing heat sources in the core (Davies and Gubbins, 2011) to estimate a maximum layer thickness of $\sim 700 \mathrm{~km}$. However, they believed that this value probably represented an overestimate as such a thick layer would likely be incompatible with observed geomagnetic secular variation.

Additional constraints can be derived from the long-term thermodynamic evolution of the core. Earth's magnetic field has existed for at least the last 3.5 Gyrs (Tarduno et al., 2010), which implies that there has been enough power available to drive the dynamo for this period. A dynamo powered solely by thermal convection cannot be sustained if the CMB heat flow is subadiabatic (e.g. Nimmo, 2015). Subadiabatic conditions can persist during inner core growth, where partitioning of light elements in the liquid drives compositional convection (Braginsky, 1963), and so the CMB heat flow must have been superadiabatic prior to inner core formation. Precipitation of $\mathrm{MgO}$ (O'Rourke and Stevenson, 2016; Badro et al., 2016) and/or $\mathrm{SiO}_{2}$ (Hirose et al., 2017) could provide additional gravitational power prior to inner core formation, which would relax the constraint on the age of thermal stratification. However, precipitation rates are still under debate (Badro et al., 2018; Du et al., 2019) and the power that is made available by precipitation depends strongly on the abundance and coupled partitioning behaviour of iron, silicon and magnesium oxides (Mittal et al., 2020). In view of these issues we do not consider precipitation in this paper.

Previous studies of Earth's core evolution have considered the timedependent growth of a thermally stable region within an adiabatic and well-mixed core. These studies solve the heat diffusion equation in the stable layer and obtain its growth from continuity conditions imposed at the interface $r_{\mathrm{s}}$ with the well-mixed interior, the basic procedure followed in this work. The studies differ primarily in their choice of boundary conditions on the diffusion equation and the numerical scheme for evolving the stable layer interface. Gubbins et al. (1982) studied thermal stratification by assuming a fixed CMB temperature and a thermal gradient at $r_{\mathrm{s}}$ fixed to the adiabatic gradient of the convective interior. They solved the time-dependent diffusion equation in the layer and included a growing inner core from the start of the run, releasing latent heat but not light elements, and obtained a $\sim 1000 \mathrm{~km}$ thick layer over 4.5 Gyrs for $k=15 \mathrm{~W} \mathrm{~m}^{-1} \mathrm{~K}^{-1}$. Labrosse et al. (1997) modelled thermal stratification as a Stefan problem, which allows both the temperature and its gradient to be continuous at $r_{\mathrm{s}}$. For a linearly decreasing CMB heat flow that fell below the adiabat around 3 Gyrs they obtained a stable layer of $\sim 600 \mathrm{~km}$ thickness at the present day, about double the rate of growth in Gubbins et al. (1982) most likely owing to the larger thermal conductivity of $60 \mathrm{~W} \mathrm{~m}^{-1} \mathrm{~K}^{-1}$. Lister and Buffett (1998) did solve for a uniform composition within the stable layer, which they argued would arise from mixing due to salt finger instabilities. They allowed jumps in both temperature and composition at $r_{\mathrm{s}}$ and evolved the interface to maintain continuity of the overall density. Using similar parameters to Labrosse et al. (1997) they found that the layer grew to just $\sim 400 \mathrm{~km}$ in nearly $3 \mathrm{Gyrs}$, due to the negative build up of compositional buoyancy slowing down the advance of the layer. Nakagawa (2018) studied thermochemical stratification driven by subadiabatic conditions and enrichment of the upper core in $\mathrm{FeO}$. He assumed steady solutions for the heat equation in the stable layer and varied $Q_{c}$ and the chemical diffusivity of $\mathrm{FeO}$ in order to match the present-day layer thickness inferred from geomagnetic secular variation. The lack of consensus regarding layer properties suggests the need for a systematic study of core evolution across a broad range of model parameters.

Thermal stratification has been considered in the cores of other terrestrial bodies. Models of Mercury's interior structure (Dumberry and Rivoldini, 2015) and dynamo (Christensen, 2006) suggest the presence of a thermally stable layer in the core, the evolution of which has been modelled using steady state solutions (Knibbe and van Westrenen, 2018). For Mars, a transition to subadiabatic conditions is usually invoked to explain the demise of a core dynamo around $4 \mathrm{Ga}$ (Stevenson, 2001; Williams and Nimmo, 2004; Davies and Pommier, 2018). The cores of Ganymede (Rückriemen et al., 2015) and the moon (Laneuville et al., 2014) are also thought to be thermally stratified at the present day. There is thus a broad utility for a general framework for modelling thermal stratification in terrestrial bodies.

In this paper we develop a new numerical code to model the growth of thermally stable layers and apply it to Earth's core. The purpose of this paper is twofold. First, we conduct a systematic parameter study in order to place constraints on the present-day thickness and strength of a thermally stable layer. We explore a wide range of input parameters including different core chemical and thermal properties and CMB heat flows and focus on high values of the thermal conductivity (de Koker et al., 2012; Pozzo et al., 2013; Gomi et al., 2013), since this favours thicker layers. Second, we consider the role of convective entrainment at the base of the layer, which has been neglected in the previous models of thermal stratification. Entrainment of buoyant fluid at the base of the stable layer can arise from downward mixing by flow in the bulk turbulent core (Turner, 1973), which acts to slow layer growth. Various parameterisations of the entrainment process have been considered and some can be shown to be equivalent (Lister, 1995). Here we implement a simple and flexible procedure that does not appeal to any specific mechanism and introduces a single 'entrainment coefficient' $E$ into the boundary conditions for the heat equation. The value of $E$ probably depends on the details of the convective dynamics within the core (Lister, 1995) and may thus vary through time. However, in view of the current incomplete understanding of the relevant processes we consider a range of constant $E$ values in this study.

This paper is organised as follows. In Section 2 we describe our evolution model of the convecting core, which follows closely the study of Davies (2015), and the new model of the thermally stable region below the CMB. Code validation is demonstrated in Section 2.3. Parameter selection, including parameterisation of the CMB heat flow, is discussed in Section 3. Results are presented in Section 4 and discussion and conclusions are presented in Section 5 .

\section{Methods}

The numerical model developed in this work consists of three main regions: the solid inner core, convecting outer core and the stable layer below the CMB (Fig. 1). The inner core boundary (ICB) is located at radius $r=r_{\mathrm{i}}(t)$, the base of the stable layer is at $r=r_{\mathrm{s}}(t)$, which varies with time $t$, and the CMB is at $r=r_{\mathrm{c}}$. For the solid and convecting regions we use the model of Davies (2015), which is based on well-established theory (Gubbins et al., 2003, 2004; Nimmo, 2015) and so only a brief overview is given. The stable layer model and its coupling to the liquid is 


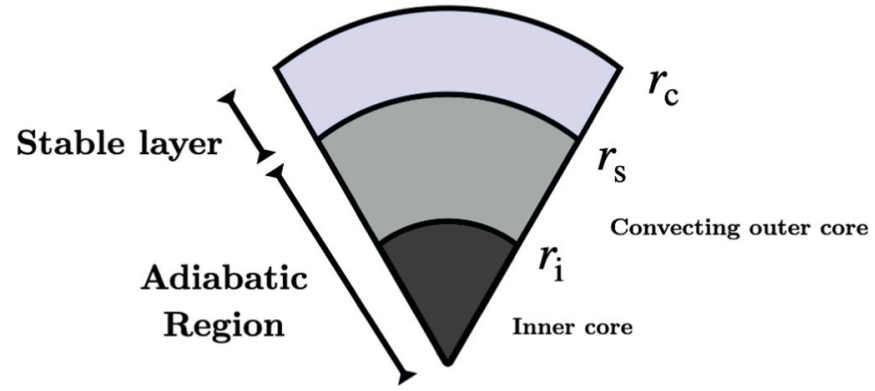

Fig. 1. 1D representation of the core. The ICB is at the radius $r_{\mathrm{i}}$, the stable layer interface at $r_{\mathrm{s}}$, and the CMB at $r_{\mathrm{c}}$. The adiabatic region is defined as $0 \leq r \leq r_{\mathrm{s}}$ and the stable layer at $r_{\mathrm{s}} \leq r \leq r_{\mathrm{c}}$.

new and will be described in detail. Heat transfer in the layer is assumed to be by conduction alone and so we verify that our code reproduces a number of standard analytical solutions.

The standard procedure for analysing core evolution over geological timescales is to average the equations governing conservation of mass, momentum and energy over timescales that are long compared to those associated with the dynamo process but short compared to the evolution timescale of the core (Braginsky and Roberts, 1995; Gubbins et al., 2003; Nimmo, 2015). In the convecting core lateral density fluctuations are thought to be much smaller than the radial density variation (Stevenson, 1987) and are assumed to average out. This assumption is also applied to the stable region, which essentially ignores effects arising from baroclinic flows driven by lateral heat flow variations at the CMB (Aubert et al., 2013; Davies and Mound, 2019). The basic state of the whole core therefore depends only on $r$ and $t$. Fluctuations of kinetic and magnetic energy are neglected and the CMB is taken to be a simple spherical interface that is electrically insulating, tractionless and impenetrable.

Core composition is constrained by the total core mass and the density difference $\Delta \rho$ between the inner and outer cores. Constraints from seismic normal modes give $\Delta \rho=800 \pm 200 \mathrm{~kg} \mathrm{~m}^{-3}$ (Masters and Gubbins, 2003) of which around $240 \mathrm{~kg} \mathrm{~m}^{-3}$ is due to the density difference between solid and liquid iron at the same pressure $P$ and temperature $T$ (Alfè et al., 2001); the rest is due to enrichment of the liquid in light elements. We use the Fe-Si-O model of Alfè et al. (2002a, see also Badro et al. (2014)) in which all O partitions into the liquid on freezing, thus matching $\Delta \rho$, while Si partitions almost evenly between liquid and
Table 2

Parameter list. The bottom half of table splits values between oxygen and silicon.

\begin{tabular}{|c|c|c|c|c|}
\hline Symbol & Meaning & \multicolumn{2}{|l|}{ Value } & Units \\
\hline$\rho^{i}$ & Inner core density & & & $\mathrm{kg} \mathrm{m}^{-3}$ \\
\hline$\rho^{o}$ & Outer core density & & & $\mathrm{kg} \mathrm{m}^{-3}$ \\
\hline$g$ & Gravity & & & $\mathrm{m} \mathrm{s}^{-2}$ \\
\hline$P$ & Pressure & & & $\mathrm{GPa}$ \\
\hline$\alpha_{T}$ & Thermal expansivity & $10^{-5}$ & & $\mathrm{~K}^{-1}$ \\
\hline \multirow[t]{2}{*}{$C_{p}$} & Specific heat capacity & 800 & & $\begin{array}{l}\mathrm{J} \mathrm{kg}^{-1} \\
\mathrm{~K}^{-1}\end{array}$ \\
\hline & & $\mathrm{O}$ & $\mathrm{Si}$ & \\
\hline$\mu_{x}^{l}-\mu_{x}^{s}$ & $\begin{array}{l}\text { Change in chemical potential from } \\
\text { liquid to solid Fe- } x\end{array}$ & -2.6 & -0.05 & $\begin{array}{l}\mathrm{eV} \\
\text { atom }^{-1}\end{array}$ \\
\hline$\lambda_{x}^{l}$ & $\begin{array}{l}\text { Linear correction to ideal solution } \\
\text { in liquid Fe- } x\end{array}$ & 3.25 & 3.6 & $\begin{array}{l}\mathrm{eV} \\
\text { atom }^{-1}\end{array}$ \\
\hline$\lambda_{x}^{s}$ & $\begin{array}{l}\text { Linear correction to ideal solution } \\
\text { in solid Fe- } x\end{array}$ & 0 & 2.7 & $\begin{array}{l}\mathrm{eV} \\
\text { atom }^{-1}\end{array}$ \\
\hline$\alpha_{x}^{c}$ & Chemical expansivity & 1.1 & 0.86 & - \\
\hline$D$ & Mass diffusivity & $10^{-8}$ & $\begin{array}{l}5 \times \\
10^{-9}\end{array}$ & $\mathrm{~m}^{2} \mathrm{~s}^{-1}$ \\
\hline $\begin{array}{l}\left(\partial \mu_{x} /\right. \\
\left.\quad \partial c_{x}^{l}\right)_{P, T}\end{array}$ & Heat of mixing & $\begin{array}{l}16 \times \\
10^{7}\end{array}$ & $\begin{array}{l}8.6 \times \\
10^{7}\end{array}$ & $\mathrm{~J}$ \\
\hline
\end{tabular}

solid cores thus matching the core mass. We consider 3 compositions defined by the molar fractions of $\mathrm{O}, \bar{c}_{\mathrm{O}}^{l}$, and $\mathrm{Si}, \bar{c}_{\mathrm{Si}}^{l}$, which are taken from Alfè et al. (2002a); Gubbins et al. (2015) and Davies et al. (2015) and are given in Table 1. Both mole and mass fractions are needed for the analysis and are related by

$c_{x}^{l / s}=\frac{A_{x}}{A} \bar{c}_{x}^{l / s}$,

where an overbar denotes a mole fraction, $A_{x}$ is the atomic mass of element $x, A$ is the mean atomic mass of the mixture, and the superscript denotes liquid or solid phase. Core temperature and transport properties are calculated self-consistently for each composition. All parameter values are listed in Tables 1 and 2.

We extend the model of Davies (2015), itself based on the work of Gubbins et al. (2003) and Gubbins (2004), to include the stable layer. Global conservation of energy through the core requires that (Gubbins, 2004; Gubbins et al., 2003; Landau and Lifshitz, 1987)

$$
\overbrace{-\oint k \nabla T \cdot \mathbf{n d} S}^{Q_{c}}=\overbrace{-\int \rho C_{p} \frac{\mathrm{d} T}{\mathrm{~d} t} \mathrm{~d} V}^{Q_{\mathrm{s}}}+\overbrace{\int \rho \psi \alpha_{x}^{\prime} \frac{\mathrm{d} c_{x}^{l}}{\mathrm{~d} t} \mathrm{~d} V_{\text {conv }}}^{Q_{\mathrm{g}}}+\overbrace{4 \pi r_{\mathrm{i}}^{2} \rho_{\mathrm{i}} L \frac{\mathrm{d} r_{\mathrm{i}}}{\mathrm{d} t}}^{Q_{\mathrm{L}}},
$$

Table 1

Parameters taken for different ICB density jumps, $\Delta \rho$. The latent heat is $T_{\mathrm{a}} \Delta S$.

\begin{tabular}{|c|c|c|c|c|c|c|}
\hline \multirow[t]{2}{*}{ Symbol } & \multicolumn{2}{|l|}{ Meaning } & \multicolumn{3}{|l|}{ Value } & \multirow{2}{*}{$\frac{\text { Units }}{\mathrm{kg} \mathrm{m}^{-3}}$} \\
\hline & $\Delta \rho$ & & 600 & 800 & 1000 & \\
\hline $\bar{c}_{O}^{l}$ & O mole fraction & & 0.08 & 0.13 & 0.17 & - \\
\hline $\bar{c}_{S i}^{l}$ & Si mole fraction & & 0.10 & 0.08 & 0.02 & - \\
\hline$c_{O}^{l}$ & O mass fraction & & 0.0256 & 0.0428 & 0.0559 & - \\
\hline$c_{S i}^{l}$ & Si mass fraction & & 0.0554 & 0.0430 & 0.0096 & - \\
\hline \multirow[t]{3}{*}{$T_{a}$} & Adiabatic temperature & $T_{1}$ & -2.17 & -5.70 & -4.44 & $10^{-9} \mathrm{~K} \mathrm{~m}^{-1}$ \\
\hline & & $T_{2}$ & -1.98 & -2.03 & -1.88 & $10^{-14} \mathrm{~K} \mathrm{~m}^{-2}$ \\
\hline & & $T_{3}$ & -6.00 & -2.12 & -7.74 & $10^{-22} \mathrm{~K} \mathrm{~m}^{-3}$ \\
\hline \multirow[t]{4}{*}{$k$} & Thermal conductivity & $k_{0}$ & 1.66 & 1.57 & 1.60 & $10^{2} \mathrm{~W} \mathrm{~m}^{-1} \mathrm{~K}^{-1}$ \\
\hline & & $k_{1}$ & 0.59 & -1.11 & -2.41 & $10^{-6} \mathrm{~W} \mathrm{~m}^{-2} \mathrm{~K}^{-1}$ \\
\hline & & $k_{2}$ & -5.25 & -4.04 & -4.04 & $10^{-12} \mathrm{~W} \mathrm{~m}^{-3} \mathrm{~K}^{-1}$ \\
\hline & & $k_{2}$ & 6.55 & -7.58 & -12.00 & $10^{-19} \mathrm{~W} \mathrm{~m}^{-4} \mathrm{~K}^{-1}$ \\
\hline \multirow[t]{4}{*}{$T_{m, F e}$} & Fe melting temperature & $T_{m_{0}}$ & 1.70 & & & $10^{3} \mathrm{~K}$ \\
\hline & & $T_{m_{1}}$ & 2.73 & & & $10^{-8} \mathrm{~K} \mathrm{~Pa}^{-1}$ \\
\hline & & $T_{m_{2}}$ & -6.65 & & & $10^{-20} \mathrm{~K} \mathrm{~Pa}^{-2}$ \\
\hline & & $T_{m_{3}}$ & 7.95 & & & $10^{-32} \mathrm{~K} \mathrm{~Pa}^{-3}$ \\
\hline \multirow[t]{5}{*}{$\Delta S$} & Entropy of melting & $\Delta S_{0}$ & 1.91 & & & $k_{b}$ \\
\hline & & $\Delta S_{1}$ & -1.19 & & & $10^{-11} k_{b} \mathrm{~Pa}^{-1}$ \\
\hline & & $\Delta S_{2}$ & 7.09 & & & $10^{-23} k_{b} \mathrm{~Pa}^{-2}$ \\
\hline & & $\Delta S_{3}$ & -1.94 & & & $10^{-34} k_{b} \mathrm{~Pa}^{-3}$ \\
\hline & & $\Delta S_{4}$ & 1.95 & & & $10^{-46} k_{b} \mathrm{~Pa}^{-4}$ \\
\hline
\end{tabular}


where $k(r)$ is thermal conductivity, $\rho(r)$ is the density, $C_{p}$ the specific heat at constant pressure, $\psi(r)$ the gravitational potential, $\alpha_{x}^{l}$ the expansion coefficient for element $x$ in the liquid phase, $L=T \Delta S_{\mathrm{Fe}}$ the latent heat coefficient with $\Delta S_{\mathrm{Fe}}$ the entropy of melting for pure iron, $V$ the volume of the whole core, and $S$ the surface of the core with outward normal $\mathbf{n}$. Subscripts i, c, rs and conv denote quantities evaluated at $r_{\mathrm{i}}, r_{\mathrm{c}}$, $r_{\mathrm{s}}$ and over the convecting core respectively. Eq. (2) states that the heat $Q_{c}$ leaving the core across the CMB is balanced by the heat sources within the core: the sensible heat $Q_{s}$, gravitational energy $Q_{g}$ released as light elements left in the liquid at the ICB mix the core, and latent heat $Q_{\mathrm{L}}$ released on freezing at the ICB. In the $Q_{\mathrm{g}}$ term there is an implied summation over the elements $x \in\{O, S i\}$. Heat of reaction and terms arising from the slow contraction of the core are small and have been neglected (Gubbins et al., 2003; Davies, 2015). We have also neglected radiogenic heating due to ${ }^{40} \mathrm{~K}$ since recent calculations suggest that only minor amounts of potassium will partition into the core (Xiong et al., 2018). Finally, since we have neglected terms associated with the slow contraction, advective derivatives in Gubbins et al. (2003) and Gubbins (2004), are simply material derivatives here.

Note that there are no new terms in Eq. (2) that arise from including a stable layer. The only difference comes from the values used to evaluate the total integrated quantities. The global energy balance can therefore be divided into contributions from the stable layer and the remainder of the core. All of the latent heat released at the ICB passes through the CMB (Davies and Gubbins, 2011). We follow Lister and Buffett (1998) by assuming that any gravitational energy change due to rearrangement of mass within the stable layer is small enough to neglect. With these assumptions $Q_{\mathrm{L}}$ and $Q_{\mathrm{g}}$ are apportioned to the energy balance of the wellmixed core and the global energy balance can be written

$Q_{\mathrm{c}}=-4 \pi \int_{r_{\mathrm{s}}}^{r_{\mathrm{c}}} r^{2} \rho C_{p} \frac{\mathrm{d} T}{\mathrm{~d} t} \mathrm{~d} r+Q_{\mathrm{rs}}$,

where $Q_{\mathrm{rs}}=-\oint k\left(r_{\mathrm{s}}\right) \nabla T\left(r_{\mathrm{s}}\right) \mathbf{n} \cdot \mathrm{d} S$ is the heat leaving the well-mixed region. The first integral in eq. (3) is evaluated using the temperature profile from the stable layer while $Q_{\mathrm{rs}}$ is evaluated from the parameterisation of the well-mixed region.

The energy budget does not contain any information about the magnetic field and therefore cannot predict if a dynamo may be sustained. Whilst a magnetic field is generated through the induction process, electric currents in the core give rise to resistive heating. This energy loss from ohmic dissipation is transferred as heat throughout the core and so does not represent any energy transfer in/out of the core. To evaluate the potential for the geodynamo to operate an entropy balance can be constructed where the ohmic dissipation does enter the equation due to being a non-reversible process. Following an equivalent approach to the energy budget, the entropy change within the core is (Gubbins, 2004)

$$
\begin{aligned}
& \overbrace{\int k\left(\frac{\nabla T}{T}\right)^{2} \mathrm{~d} V}^{E_{\mathrm{k}}}+\overbrace{\int \frac{i^{2}}{\alpha_{x}^{D} T} \mathrm{~d} V}^{E_{\alpha}}+\overbrace{\int \frac{\Phi}{T} \mathrm{~d} V}^{E_{\mathrm{J}}} \\
& =-\underbrace{\int\left(\frac{1}{T_{\mathrm{c}}}-\frac{1}{T}\right) \rho C_{p} \frac{\mathrm{d} T}{\mathrm{~d} t} \mathrm{~d} V}_{E_{\mathrm{s}}}+\underbrace{\left(\frac{1}{T_{\mathrm{c}}}-\frac{1}{T_{\mathrm{i}}}\right) Q_{\mathrm{L}}}_{E_{\mathrm{L}}}+\underbrace{\frac{Q_{\mathrm{g}}}{T_{\mathrm{c}}}}_{E_{\mathrm{g}}}
\end{aligned}
$$

where $T_{\mathrm{i}}$ is the ICB temperature, $T_{\mathrm{c}}$ is the CMB temperature, $i^{2}$ is the square of the mass flux vector, and $\alpha_{x}^{D}$ is the barodiffusion coefficient for element $x$ given by

$\alpha_{x}^{D}=\frac{\rho D_{x}}{\left(\partial \mu_{x} / \partial c_{x}^{l}\right)_{P, T}}$,

where $D_{x}$ and $\mu_{x}$ are the molecular diffusivity and chemical potential for element $x$. The right-hand side of eq. (4) gives the rate of change of entropy, which contains contributions due to secular cooling $E_{\mathrm{s}}$, latent heat $E_{\mathrm{L}}$, and gravitational energy $E_{\mathrm{g}}$. The left-hand side gives the positive sources of entropy due to thermal conduction $E_{\mathrm{k}}$, barodiffusion $E_{\alpha}$, and the combined ohmic and viscous dissipation $E_{\mathrm{J}}$. In the geodynamo viscous dissipation is thought to be negligible (Jones, 2015) and so we hereafter take $\Phi$ to represent the ohmic heating only. $E_{\mathrm{J}}$ represents the average dissipation due to work done by the Lorentz force on the flow and can be calculated from eq. (4) once all other terms are known. The requirement $E_{\mathrm{J}}>0$ places a useful constraint on the thermal evolution of the core since observations of Earth's internally generated magnetic field date back to at least 3.5 Ga (Tarduno et al., 2010), and hence the ohmic dissipation should be positive during that period.

Following the procedure applied to the energy balance, $E_{\mathrm{s}}, E_{\mathrm{k}}$, and $E_{\alpha}$ are evaluated across both the stable and well-mixed regions using the appropriate temperature profiles while $E_{\mathrm{L}}$ can be evaluated using information from the convecting region and the $\mathrm{CMB}$ temperature. The ohmic dissipation $E_{\mathrm{J}}$ is calculated as the remainder of eq. (4) once all other terms have been evaluated. The evaluation of these terms is now described for the well-mixed and stable regions.

\subsection{Solid and liquid cores}

The basic state of the liquid and solid cores are assumed to average to an isentropic, compositionally uniform, and hydrostatic state (Braginsky and Roberts, 1995; Gubbins et al., 2004). Deviations from these radial profiles in the solid inner core are insignificant when considering global balances (Labrosse et al., 2001). In this state the core temperature $T_{\mathrm{a}}$ follows an adiabat, given by

$T_{\mathrm{a}}(r)=T_{\mathrm{cen}} \exp \left(-\int_{0}^{r} \frac{g \gamma}{\phi} \mathrm{d} r\right)$,

where $T_{\text {cen }}$ is the temperature at the center of the core, $\gamma$ is the Grüneisen parameter, $\phi$ is the seismic parameter and $g$ is gravity. The total heat flow down an adiabatic temperature gradient at the CMB is

$Q_{\mathrm{a}}=-\left.4 \pi r_{\mathrm{c}}^{2} k \frac{\partial T_{\mathrm{a}}}{\partial r}\right|_{r=r_{\mathrm{c}}}$

which, along with $Q_{c}$ determines the onset of thermal stratification. The exponential in eq. (6) varies slowly in time (Gubbins et al., 2003) and hence

$\frac{1}{T_{\mathrm{a}}} \frac{\mathrm{d} T_{\mathrm{a}}}{\mathrm{d} t}=\frac{1}{T_{\text {cen }}} \frac{\mathrm{d} T_{\text {cen }}}{\mathrm{d} t}$

to a very good approximation. This equation relates the cooling rate at any radius in the adiabatic region to the cooling rate at the centre of the core. Here it is convenient to take the reference point as the centre rather than the CMB as in Davies (2015) since the adiabatic region does not extend to the top of the core.

The contributions from the well-mixed region to all terms on the right-hands side of eqs. (2) and (4) can be expressed in terms of the cooling rate at the centre, $\mathrm{d} T_{\text {cen }} / \mathrm{d} t$. The rate of change of the inner core radius is given by (Gubbins et al., 2003)

$\frac{\mathrm{d} r_{\mathrm{i}}}{\mathrm{d} t}=\frac{1}{\left(\mathrm{~d} T_{\mathrm{m}} / \mathrm{d} r\right)_{r=r_{\mathrm{i}}}-\left(\mathrm{d} T_{\mathrm{a}} / \mathrm{d} r\right)_{r=r_{\mathrm{i}}}} \frac{T_{\mathrm{i}}}{T_{\text {cen }}} \frac{\mathrm{d} T_{\text {cen }}}{\mathrm{d} t}=C_{r} \frac{\mathrm{d} T_{\text {cen }}}{\mathrm{d} t}$,

where $T_{\mathrm{m}}$ is the melting temperature of the core alloy. This equation defines the quantity $C_{r}$, which relates the core cooling rate to the inner core growth rate. The rate of change of light element $x$ in the liquid is obtained from conservation of mass and is (Gubbins et al., 2004)

$\frac{\mathrm{d} c_{x}^{l}}{\mathrm{~d} t}=\frac{4 \pi r_{\mathrm{i}}^{2} \rho_{\mathrm{i}}\left(c_{x}^{s}-c_{x}^{l}\right)}{M_{\text {conv }}} \frac{\mathrm{d} r_{\mathrm{i}}}{\mathrm{d} t}=C_{x}^{l} \frac{\mathrm{d} r_{\mathrm{i}}}{\mathrm{d} t}$,

where $M_{\text {conv }}$ is the mass of the convecting core.

With the above definitions the energy balance for the well-mixed 
region can be written

$Q_{\mathrm{rs}}=-\frac{C_{p}}{T_{\text {cen }}} \frac{\mathrm{d} T_{\text {cen }}}{\mathrm{d} t} \int \rho T_{\mathrm{a}} \mathrm{d} V_{\mathrm{s}}+\sum_{x} \alpha_{x}^{l} C_{r} C_{x}^{l} \frac{\mathrm{d} T_{\text {cen }}}{\mathrm{d} t} \int \rho \psi \mathrm{d} V_{\text {conv }}+4 \pi r_{\mathrm{i}}^{2} \rho_{\mathrm{i}} L C_{r} \frac{\mathrm{d} T_{\text {cen }}}{\mathrm{d} t}$

or

$Q_{\mathrm{rs}}=\frac{d T_{c e n}}{d t}\left(\widetilde{Q}_{s}+\widetilde{Q}_{g}+\widetilde{Q}_{l}\right)$

where $V_{\mathrm{s}}(t)$ is the volume of the core below $r_{\mathrm{s}}(t)$. If no stable layer exists, $Q_{\mathrm{rs}}=Q_{\mathrm{c}}$ and $r_{\mathrm{s}}=r_{\mathrm{c}} \cdot Q_{\mathrm{rs}}$ is either known based on the temperature profile at the base of the stable layer or from a constraint on the CMB heat flow so eq. (12) may be numerically integrated to solve for $T_{\text {cen }}$.

All radially varying parameters are calculated on a uniform grid and numerically integrated with the trapezoid rule. The radial variation in $T_{\mathrm{a}}$, the melting temperature of pure iron $T_{\mathrm{m}, \mathrm{Fe}}$, the entropy of melting $\Delta S_{\mathrm{Fe}}$, thermal conductivity $k$ and density $\rho$ are expressed by polynomials in the form:

$T_{\mathrm{a}}(r)=T_{c e n}\left(1+T_{1} r+T_{2} r^{2}+\ldots T_{N} r^{N}\right)$,

$T_{\mathrm{m}, \mathrm{Fe}}(P)=T_{m_{0}}+T_{m_{1}} P+T_{m_{2}} P^{2}+\ldots T_{m_{N}} P^{N}$,

$\Delta S_{\mathrm{Fe}}(P)=\Delta S_{0}+\Delta S_{1} P+\Delta S_{2} P^{2}+\ldots \Delta S_{N} P^{N}$.

$\rho(r)=\left(\begin{array}{l}\rho_{0}^{i}+\rho_{1}^{i} r+\rho_{2}^{i} r^{2}+\ldots \rho_{N}^{i} r^{N} \quad \text { for } r \leq r_{i} \\ \rho_{0}^{o}+\rho_{1}^{o} r+\rho_{2}^{o} r^{2}+\ldots \rho_{N}^{o} r^{N} \quad \text { for } r_{i} \leq r \leq r_{\mathrm{s}}\end{array}\right.$

For $\rho$ the polynomial coefficients are all assumed constant in time with the exception of $\rho_{0}^{o}$ which is adjusted to ensure mass is conserved as the inner core radius changes. $g(r)$ and $\psi(r)$ are found by integrating the density polynomials where $g(0)=0$ and $\psi\left(r_{\mathrm{c}}\right)=0$. The pressure $P(r)$ is found by numerically integrating the hydrostatic pressure gradient $\mathrm{d} P$ / $\mathrm{d} r=-\rho g$, subject to a specified CMB pressure of $135 \mathrm{GPa}$.

The melting temperature $T_{\mathrm{m}}$ of the core alloy is written as

$T_{\mathrm{m}}=T_{\mathrm{m}, \mathrm{Fe}}+\sum_{x} \Delta T_{\mathrm{x}}$

where $\Delta T_{\mathrm{x}}$ is the depression of the melting point by impurity $x$ and we have assumed that each light element alters the melting temperature independently. $\Delta T_{\mathrm{x}}$ is taken from the theory of Alfè et al. (2002b) and is written

$\Delta T_{\mathrm{x}}=\frac{T_{\mathrm{m}, \mathrm{Fe}}}{\Delta S_{\mathrm{Fe}}}\left(\bar{c}_{x}^{s}-\bar{c}_{x}^{l}\right)$,

where $\bar{c}_{x}^{s}$ is the mole fraction of element $x$ in the solid. Relating $\bar{c}_{x}^{s}$ and $\bar{c}_{x}^{l}$ requires knowledge of how light elements partition between the liquid and solid as the inner core grows. We follow Alfè et al. (2002a) to express equality of the chemical potentials as

$\mu_{0}^{l}+\lambda_{x}^{l} \bar{c}_{x}^{l}+k_{b} T_{\mathrm{m}} \ln \left(\bar{c}_{x}^{l}\right)=\mu_{0}^{s}+\lambda_{x}^{s} \bar{c}_{x}^{s}+k_{b} T_{\mathrm{m}} \ln \left(\bar{c}_{x}^{s}\right)$,

where $\mu_{0}^{s / l}$ is the reference chemical potential in either the solid or liquid, $\lambda$ represents a linear correction to the chemical potentials to account for deviations from an ideal solution and $k_{b}$ is the Boltzmann constant. Substituting eqs. (17) and eqs. (18) yields a transcendental equation for $\bar{c}_{x}^{s}$ that can be solved using the bisection method. Mass and molar fractions are related by eq. (1).

The adiabatic temperature profile is calculated at each timestep and its gradient $\mathrm{d} T_{\mathrm{a}} / \mathrm{d} r$ is used to calculate the stable layer evolution. If no stable layer is present, $E_{\mathrm{J}}$ is directly calculated at this stage by eq. (4). Inner core nucleation occurs when $T_{\mathrm{a}}(r=0)=T_{\mathrm{m}}(r=0)$ and $r_{\mathrm{i}}$ is thereafter defined as the radius where $T_{\mathrm{a}}(r)=T_{\mathrm{m}}(r)$. We assume that the core solidifies from the inside out and hence the radial gradient in the melting temperature is necessarily steeper than the adiabat.

\subsection{Stable layer: Theory}

Within the stable layer we assume that heat transport is governed by thermal conduction:

$\rho_{s} C_{p} \frac{\partial T_{\mathrm{s}}}{\partial t}=\nabla \cdot\left(k \nabla T_{\mathrm{s}}\right)$

where $\rho_{s}$ and $T_{\mathrm{s}}$ are the density and temperature in the stable layer and the thermal conductivity $k$ is allowed to vary with radius. Composition is assumed to have a uniform value and not to contribute to any time evolution of the stable layer. The impact of this assumption upon our results, in particular in the context of a growing inner core enriching the well-mixed region with light element, is discussed further in Section 5.

In order to solve eq. (20), we first need to consider that this is a moving boundary problem, since $r_{\mathrm{s}}$ is time dependent. We will require an additional condition at $r_{\mathrm{s}}$ in order to solve for the motion of the interface. We will treat the domain as fixed, solving for the temperature given 2 flux boundary conditions, one at $r_{\mathrm{c}}$ and one at $r_{\mathrm{s}}$. We then determine the required change in $r_{\mathrm{s}}$ to satisfy continuity of temperature, therefore specifying 2 conditions in total at $r_{\mathrm{s}}$. We assume no mass flux at the $\mathrm{CMB}$ and so the thermal gradient at the $\mathrm{CMB}$ is:

$\left(\frac{\partial T_{\mathrm{s}}}{\partial r}\right)_{r_{\mathrm{c}}}=-\frac{Q_{\mathrm{c}}(t)}{4 \pi r_{\mathrm{c}}^{2} k_{c}}$

At the time-dependent stable layer interface, $r_{\mathrm{s}}(t)$, the situation is more complicated. Dynamical instabilities arising from penetrative convection or shear flows may promote mixing across the interface (Turner, 1973). Entrainment of fluid from the stable region into the wellmixed interior will limit the growth of the layer, either slowing it down or eroding it altogether. Following Lister (1995) we assume that these processes arise in a thin mixing layer that sits between the convecting bulk and the stable layer in which the temperature changes continuously from the adiabatic interior to the conductive profile in the stable layer. In the parameterised model the thickness of the mixing layer is neglected and its effect appears in the boundary condition at $r_{\mathrm{s}}$ using the formulation of Lister (1995):

$\frac{\partial T_{\mathrm{s}}}{\partial r}=(1-E) \frac{\partial T_{\mathrm{a}}}{\partial r} \quad$ at $\quad r=r_{\mathrm{s}}(t)$

where $E$ is the entrainment coefficient. Both upper and lower boundary conditions are therefore of the Neumann type.

A Crank-Nicolson scheme is used to solve the diffusion equation with temperature computed on a radial grid with an even spacing $\Delta r$ across the layer. The Crank-Nicolson method is second order accurate and is unconditionally stable for diffusion problems. As the size of the domain changes so does the total number of nodes to keep the same resolution and linear interpolation is used to regrid. For accuracy of the CrankNicolson scheme the CFL number should satisfy

$0.5 \geq \frac{\kappa \Delta t}{2 \Delta r^{2}}$

where $\Delta t$ is the timestep. If this condition is not satisfied by the current $\Delta t$ then a smaller timestep is used and the iteration is repeated until eq. (23) is satisfied.

At time $t$ the evolution of the convecting layer is first determined in the fixed region $0 \leq r \leq r_{\mathrm{s}}(t)$ before the stable layer is evolved using eq. (20) in the fixed region $r_{\mathrm{s}}(t) \leq r \leq r_{\mathrm{c}}$. To solve eq. (20) the upper boundary condition eq. (21) is calculated from the imposed CMB heat flux while $\partial T_{\mathrm{a}} / \partial r$ in eq. (22) is obtained from the solution of the energy eq. (12) in the convecting region at the current timestep. The density in (20) is derived from the temperature in the stable layer at the previous iteration as 
$\rho_{s}=\rho\left[1-\alpha_{T}\left(T_{\mathrm{s}}-T_{\mathrm{a}}\right)\right]$,

where $\rho$ and $T_{\mathrm{a}}$ are respectively the PREM density and adiabatic temperature extrapolated through the stable layer from the convecting region.

At this point the adiabatic and stable layer temperatures at the new time, $T_{\mathrm{a}}(r, t+\Delta t)$ and $T_{\mathrm{s}}(r, t+\Delta t)$, will in general be discontinuous at $r_{\mathrm{s}}(t)$, which will no longer be the point of neutral stability (Fig. 2). The new value of $r_{\mathrm{s}}(t+\Delta t)$ is obtained by checking the dynamical stability of the new thermal profile throughout the stable layer. Fluid parcels at radius $r$ are convectively unstable if (Gubbins and Roberts, 1987)

$\left|\frac{\partial T_{\mathrm{a}}(r, t+\Delta t)}{\partial r}\right|>\left|\frac{\partial T_{\mathrm{s}}(r, t+\Delta t)}{\partial r}\right|$

If fluid at any radius within the layer satisfies eq. (25) or is more dense than a fluid parcel from the adiabatic region would be when raised to its level $\left(\rho_{s}(r, t+\Delta t)>\rho(r, t+\Delta t)\right)$ then the unstable fluid is assumed to mix into the bulk; the layer thickness decreases and $r_{\mathrm{s}}(t+\Delta t)$ is moved to the point of neutral stability, $\partial T_{\mathrm{a}}(r, t+\Delta t) / \partial r=\partial T_{\mathrm{s}}(r, t+\Delta t) / \partial r$. If the entire stable layer satisfies eq. (25) then the stable region thickens and $r_{\mathrm{s}}(t+\Delta t)$ is set as the radius where $T_{\mathrm{a}}(r, t+\Delta t)=T_{\mathrm{s}}(r, t+\Delta t)$ (Fig. 2).

To obtain the temperature between $r_{\mathrm{s}}(t)$ and $r_{\mathrm{s}}(t+\Delta t)$ we linearly interpolate between $T_{\mathrm{a}}(r, t+\Delta t)$ and $T_{\mathrm{s}}(r, t+\Delta t)$. Consequently the temperature profile across the core at the end of each iteration will be continuous, but the temperature gradient will only be piecewise continuous at $r_{\mathrm{s}}(t+\Delta t)$. Since the individual layers generally cool by only a fraction of a degree over a timestep of 1 million years the discontinuity in $\partial T / \partial r$ is orders of magnitude smaller than the absolute temperature gradient. We have investigated different interpolation schemes that allow continuity of $T$ and $\partial T / \partial r$ at $r_{\mathrm{s}}$, however these higher order schemes generally permit unphysical behaviour such as unstable gradients in the stable region. Below we show that our code satisfactorily reproduces the results of Labrosse et al. (1997) as expected given the same choice of boundary conditions, despite different methods for evolving $r_{\mathrm{s}}$.

\subsection{Code validation}

Here we show that the diffusion code matches analytical solutions and that the stable layer evolution reproduces expected behaviour. For constant diffusivity $\kappa=k /\left(\rho C_{p}\right)$ we consider analytical solutions for the cases of fixed temperature and fixed temperature gradient at the outer boundary of a full sphere. For both cases the initial condition is taken to be a uniform temperature, $T_{1}$, and the temperature gradient at $r=0$ is zero. The time-dependent solution for a fixed temperature, $T_{0}$, at the outer boundary $r=a=1$ is (Crank, 1979, eq. 6.18)

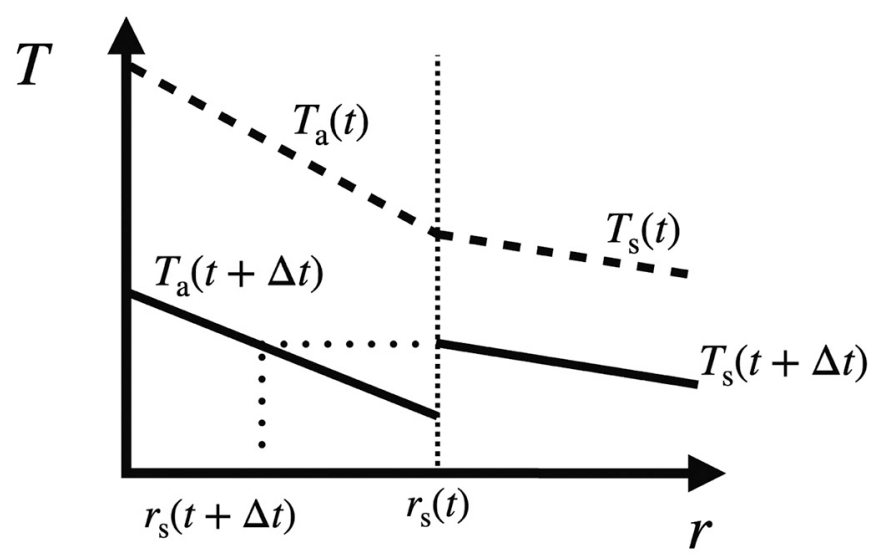

Fig. 2. Temperature profiles $T_{\mathrm{a}}$ and $T_{\mathrm{s}}$ for the adiabatic and diffusive regions at time $t$ and $t+\Delta t$. The adiabatic and stable regions are evolved independently, after which the layer interface advances to maintain continuity of temperature.
$\frac{T-T_{1}}{T_{0}-T_{1}}=1+\frac{2 a}{\pi r} \sum_{n=1}^{\infty} \frac{(-1)^{n}}{n} \sin \left(\frac{n \pi r}{a}\right) \exp \left(-\frac{\kappa n^{2} \pi^{2} t}{a^{2}}\right)$

and the solution for a fixed temperature gradient at $r=a$ is (Crank, 1979, eq. 6.45)

$T_{0}-T=-a\left(\frac{\partial T}{\partial r}\right)_{r=a}\left[\frac{3 \kappa t}{a^{2}}+\frac{1}{2} \frac{r^{2}}{a^{2}}-\frac{3}{10}-2 \frac{a}{r} \sum_{n=1}^{\infty} \frac{\sin \left(\alpha_{n} r\right)}{\alpha_{n}^{2} a^{2} \sin \left(\alpha_{n} a\right)} \exp \left(-\kappa \alpha_{n} t\right)\right]$

where $\alpha_{n}$ are defined by the $n^{\text {th }}$ root of $a \alpha_{n} \cot \left(a \alpha_{n}\right)=1$.

Numerical solutions were run in a spherical shell with $0.001 \leq r \leq a$ $=1$ to avoid the singularity at the origin, which we found to adequately represent the full-sphere geometry appropriate for the analytical solutions. Fig. 3a shows excellent agreement between the computed and analytical instantaneous temperature profile. For the parameter choice used here, only 10 radial grid points are required for the error to drop below $0.5 \%$ for both boundary condition types, showing rapid convergence (Fig. 3b).

Analytical solutions also exist for a steady state with a radially varying diffusivity. For a spherical shell with inner and outer surfaces at $r_{1}$ and $r_{2}$ which are held at constant temperature $T_{1}$ and $T_{2}$ respectively, the steady state solution takes the form (Crank, 1979, eq. 9.18)

$\frac{T_{1}-T}{T_{1}-T_{2}}=\frac{I\left(r_{1}\right)-I(r)}{I\left(r_{1}\right)-I\left(r_{2}\right)}$

where $I\left(r_{1}\right)$ and $I\left(r_{2}\right)$ are the values of the integral $I(r)$ at $r_{1}$ and $r_{2}$ given by

$I(r)=\int_{r_{1}}^{r} \frac{\mathrm{d} r}{r^{2}(1+f(r))}$,

and $\kappa$ varies in radius such that

$\kappa(r)=\kappa_{0}(1+f(r))$

Fig. 3c and d compare numerical and analytical solutions for 3 cases with $\kappa_{0}=1 \mathrm{~m}^{2} \mathrm{~s}^{-1}$ and $f(r)=0, f(r)=r$, and $f(r)=10-r$. The solution is calculated for $r_{1}=1 \mathrm{~m}, r_{2}=10 \mathrm{~m}, T_{1}=2 \mathrm{~K}$ and $T_{2}=1 \mathrm{~K}$. Good agreement is shown between numerical and analytical solutions.

We consider two cases to demonstrate the behaviour of the thermal history model with a stable layer. The equilibrium configuration in which the layer ceases to grow is obtained when the heat entering and leaving the layer are balanced: $Q_{r_{s}}=Q_{c}$. In general, the approach to this state is hindered because both $Q_{r_{s}}$ and $Q_{c}$ vary in time, so for demonstration we set constant total and adiabatic heat flows at the CMB to $Q_{\mathrm{c}}$ $=11 \mathrm{TW}$ and $Q_{\mathrm{a}}=15 \mathrm{TW}$ respectively and $\mathrm{d} T_{a} / \mathrm{d} t=0$ in the adiabatic interior, which requires that the adiabatic heat flow at all radii is also constant in time. Other parameters are $k=100 \mathrm{~W} \mathrm{~m}^{-1} \mathrm{~K}^{-1}, \kappa=10^{-6} \mathrm{~m}^{2}$ $\mathrm{s}^{-1}$ and the adiabatic gradient corresponding to $\Delta \rho=800 \mathrm{~kg} \mathrm{~m}^{-3}$ (Table 1). Fig. 4 shows how the layer quickly grows and then converges to the radii at which $Q_{r_{\mathrm{s}}}=Q_{\mathrm{c}}$. The temperature profile in the layer is elevated above the adiabat until it merges with the adiabat at $r_{\mathrm{s}}$ as expected.

Finally, we reproduce the results of Labrosse et al. (1997). We parameterise their CMB heat flow in the form

$Q_{\mathrm{c}}=\left(q_{0}+\beta t\right) \times 4 \pi r_{\mathrm{c}}^{2}$,

where $q_{0}=75 \mathrm{~mW} \mathrm{~m}^{-2}$ and $\beta=-3.5 \mathrm{~W} \mathrm{~m}^{-2} \mathrm{~s}^{-1}$. The thermal conductivity of the core is $60 \mathrm{~W} \mathrm{~m} \mathrm{~m}^{-1} \mathrm{~K}^{-1}$ and the thermal diffusivity is $5.8 \times 10^{-6} \mathrm{~m}^{2} \mathrm{~s}^{-1}$. The model matches the values of $r_{\mathrm{i}}$ and $r_{\mathrm{s}}$ of Labrosse et al. (1997) within $5 \%$ over most of the model evolution, producing a purely thermal stable layer of around $600 \mathrm{~km}$ thickness over the last 1.5 Gyrs (Fig. 5). The match to $r_{\mathrm{i}}$ is poorest near the start of the run because inner core nucleation occurs at slightly different times in the two cases. The agreement is very good considering that different methods were 

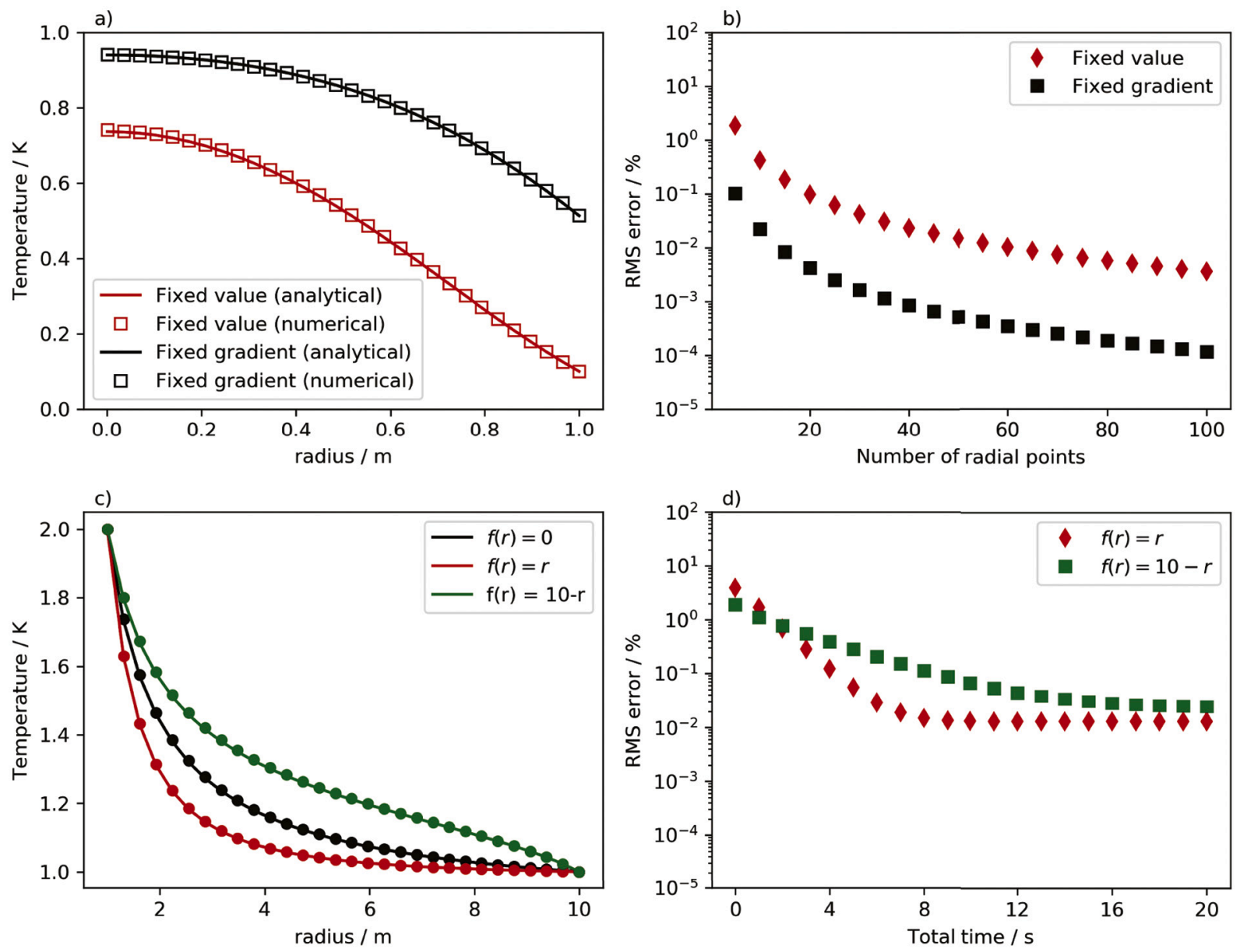

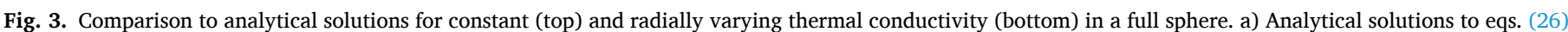

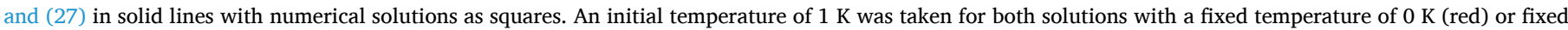

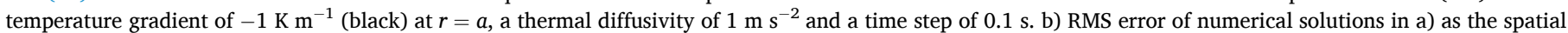

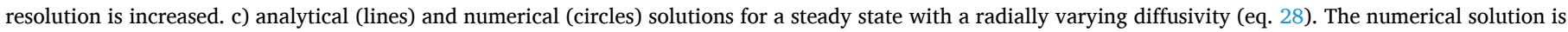

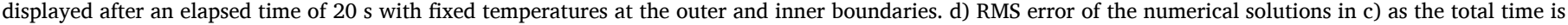

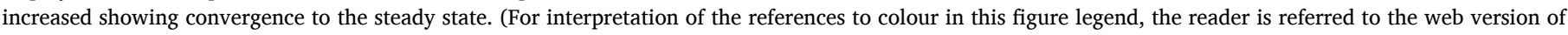
this article.)

used to model both the adiabatic interior, stable region and the evolution of the interface; these variations explain the small differences between the two cases.

\section{Parameter selection and CMB heat flow}

We consider three different sets of parameters describing core physical properties, which are taken from Davies et al. (2015) where more details can be found. Parameter sets correspond to the values of the ICB density jump $\Delta \rho=600,800$ and $1000 \mathrm{~kg} \mathrm{~m}^{-3}$. For $\Delta \rho=600$ and 800 $\mathrm{kg} \mathrm{m}^{-3}$ the corresponding $\mathrm{Si}$ and O compositions are taken from Gubbins et al. (2004), while for $\Delta \rho=1000 \mathrm{~kg} \mathrm{~m}^{-3}$ the compositions come from Gubbins et al. (2015). Note that these compositions also match the overall mass of the core. For each composition we determine the melting point depression at the ICB using eq. (18), which provides the anchor point for the adiabatic temperature. Finally, thermal conductivity was calculated by Pozzo et al. (2013) at specific points on these three adiabats. The polynomial coefficients for $T_{\mathrm{a}}, T_{\mathrm{m}}, \mathrm{Fe}$ and $k$ for the three cases are given in Table 1 .

A number of parameters are fixed in all cases, which are listed in Table 2 . The density $\rho$ in the solid inner core and convecting part of the liquid core is represented by second order polynomials with coefficients taken from PREM. These polynomials are used to analytically compute the gravity $g$, gravitational potential $\psi$ and pressure $P$. The polynomial coefficients for $\rho$ and the entropy of melting $\Delta S_{\mathrm{Fe}}$ are as in Davies (2015). The latent heat is calculated at the ICB using the polynomial representations of $T_{\mathrm{a}}$ and $\Delta S_{\mathrm{Fe}}$. The chemical properties of $\mathrm{O}$ and $\mathrm{Si}$ are taken from Gubbins et al. (2004) and are the same as those in Davies (2015).

When the Rossby number is small, convective entrainment is also expected to be small (Lister and Buffett, 1998; Levy and Fernando, 2002; Wang, 2003) and so we focus upon the case when $E=0$, giving a continuous temperature gradient throughout the core. Degrees of entrainment of chemically light plumes into thermal stratification is not so well understood. (Bouffard et al., 2020) conduct hydrodynamic simulations of erosion into a chemically stable layer, finding it to be small; however, light element impacts the density of fluid more than temperature $\left(\alpha_{c} / \alpha_{T} \approx 10^{5}\right)$. We therefore also perform simulations at $E$ $>0$ in order to quantify the impact entrainment has upon the model and to relate to potential future studies that are able to quantify entrainment rates of chemically enriched plumes into thermal stratification.

The final input to the model is the CMB heat flow $Q_{c}$. Strictly, $Q_{c}$ should be determined simultaneously with the evolving core temperature using time-dependent dynamical models of mantle convection (e.g. Nakagawa and Tackley, 2007); however, this is very time-consuming and does not allow a systematic exploration of parameter space. Another strategy is to employ a parameterised model of mantle convection (e.g. Nimmo et al., 2004; Driscoll and Bercovici, 2014), which enables self-consistent calculation of $Q_{\mathrm{c}}$ and $T_{\mathrm{c}}$ but at the expense of 
a)

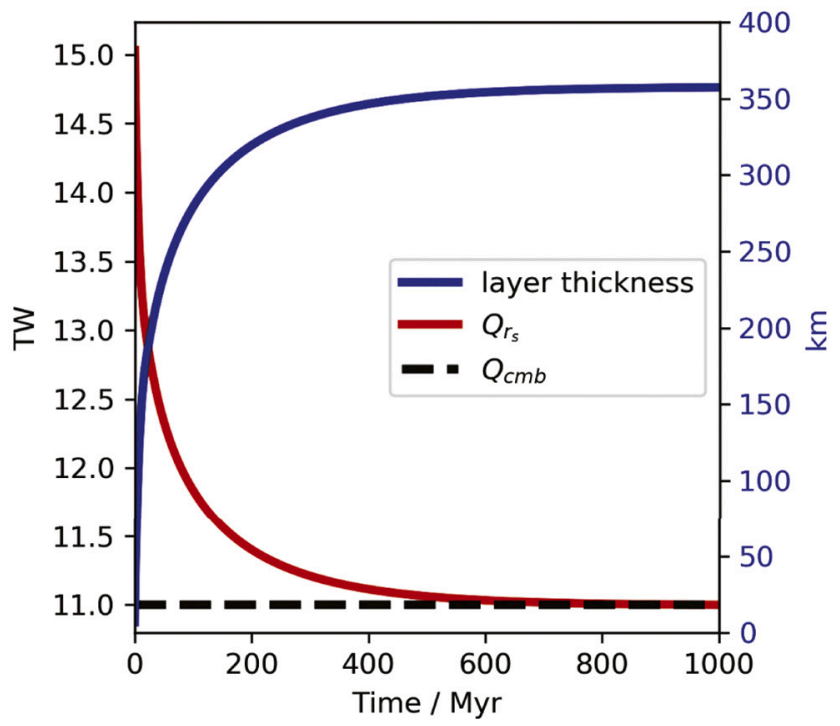

b)

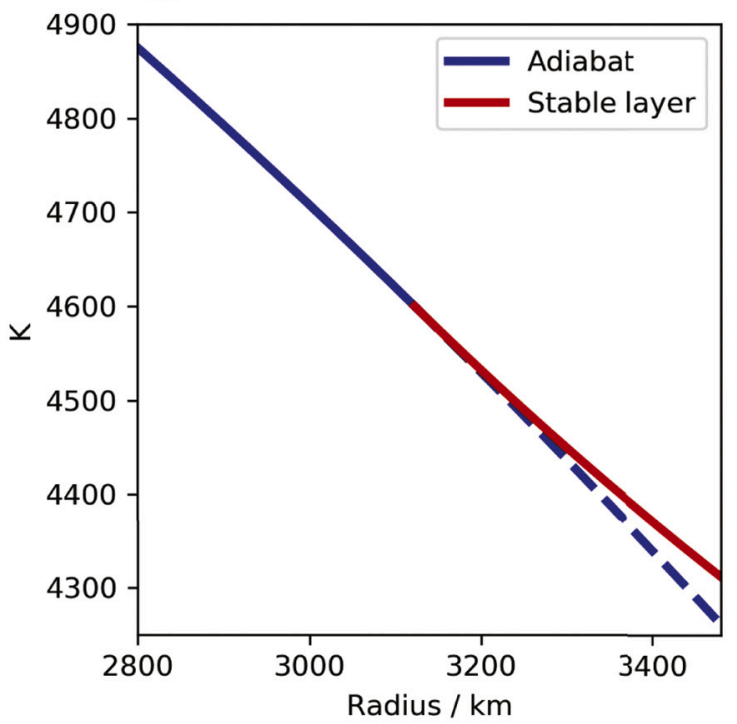

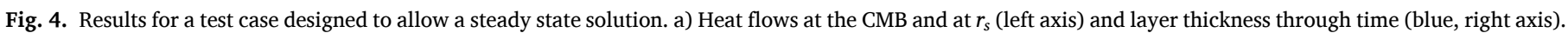

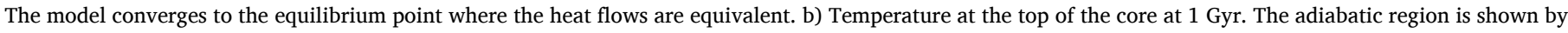

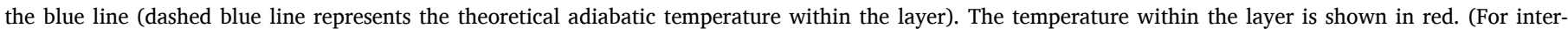
pretation of the references to colour in this figure legend, the reader is referred to the web version of this article.)
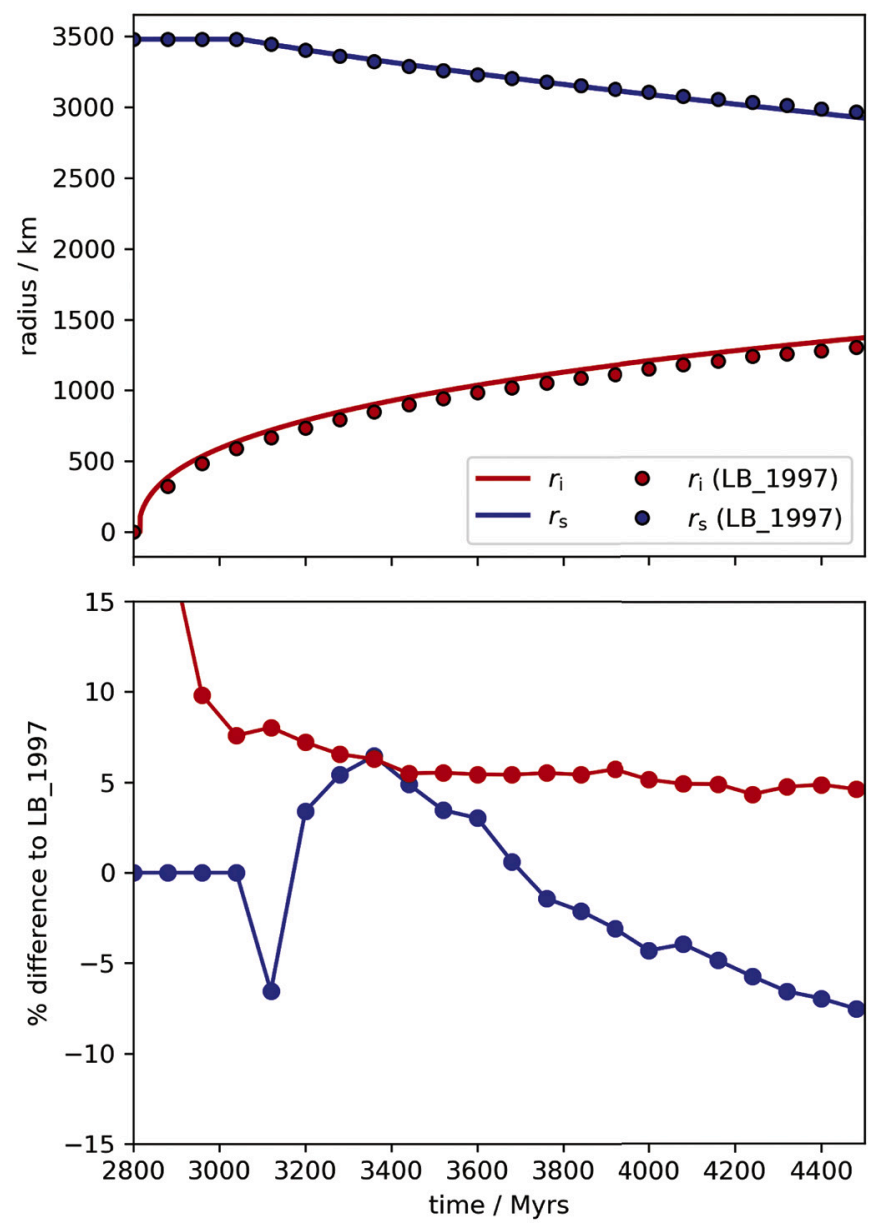

Fig. 5. Results for a test case (solid lines) matching the results of Labrosse et al. (1997) (circles). The inner core radius, $r_{\mathrm{i}}$ is shown in red and the stable layer interface, $r_{s}$, is shown in blue. (For interpretation of the references to colour in this figure legend, the reader is referred to the web version of this article.) introducing uncertain parameters such as the conductivity and viscosity of the upper and lower mantle thermal boundary layers. Moreover, a number of alternative parameterisations are available (e.g. Conrad and Hager, 1999; O'Rourke et al., 2017), which can significantly change the predicted heat flows. Fig. 6 shows time-series of $Q_{c}$ from 2 recent parameterised mantle models (Driscoll and Bercovici, 2014; Patočka et al., 2020) and the 3D mantle convection model of Nakagawa and Tackley (2015). These calculations were chosen as they used high core conductivity values of $k\left(r_{\mathrm{c}}\right) \sim 80-120 \mathrm{~W} \mathrm{~m}^{-1} \mathrm{~K}^{-1}$, produced thermal histories that match the current ICB radius, and produced enough entropy to sustain the magnetic field for the last 3.5 Gyrs. While there are significant differences between the individual heat flows, they all show an increase in $Q_{c}$ back to the early Earth $(<3.5 \mathrm{Ga})$ and can be reasonable represented with a linear trend in more recent times.

The objective of this study is to constrain the range of thermal stable layer properties that are consistent with current knowledge of the coremantle system and so we attempt to consider as wide a range of $Q_{c}$ as possible. On time scales comparable to the inner core age (0.5-1 Gyrs) that are of interest, all results in Fig. 6 are linear to a good approximation. Results presented here are related to the longer term trend back to $3.5 \mathrm{Ga}$ as discussed in sections 4 and 5 . We are therefore motivated to write $Q_{c}$ using a simple linear equation, which allows us to systematically sample a large range of solutions. We write

$Q_{\mathrm{c}}(t)=A+B(4.5 \mathrm{Gyrs}-t)$,

where $A$ and $B$ are the present day CMB heat flow and the linear decrease in $Q_{\mathrm{c}}$ over time. The best fit linear decrease in $Q_{\mathrm{c}}$ over the last $0.7 \mathrm{Gyrs}$ for the histories shown in Fig. 6 give $B$ values of 2.8, 1.6, and 2.3 TW $\mathrm{Gyr}^{-1}$ for the calculations of Patočka et al. (2020), Nakagawa and Tackley (2015), and Driscoll and Bercovici (2014), respectively. We will show that such low $B$ values produce present-day stable layers of $\sim 100$ $\mathrm{km}$ or less. We therefore focus on values of $B$ in the range 1-13 $\mathrm{TW} \mathrm{Gyr}^{-1}$ in order to sample the extreme conditions that may produce layers of $100-400 \mathrm{~km}$ as suggested by recent studies (Kaneshima, 2017).

The main disadvantage of this approach, i.e. that $Q_{c}$ does not respond to changes in core temperature, can be mitigated by considering a range of different initial core temperatures. However, the thermostat effect provided by the strong dependence of mantle viscosity on temperature 


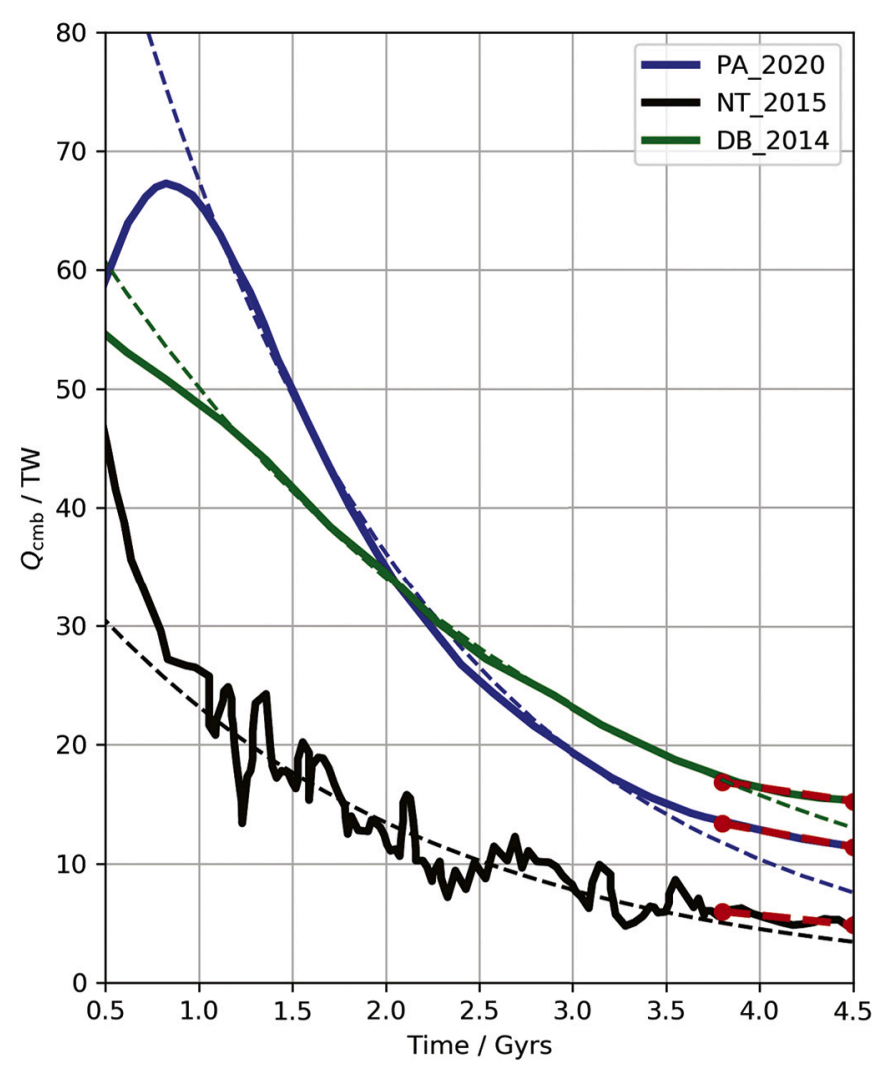

Fig. 6. Published CMB heat flows from Patočka et al. (2020) (PA2020), Driscoll and Bercovici (2014) (DB2014), and Nakagawa and Tackley (2015) (NT2015). PA2020 used a viscosity constrast across the mantle of 5, with an activation energy of $300 \mathrm{~kJ} \mathrm{~mol}^{-1}$ as shown on their fig. 12. DB2014 is from their Earth case as shown in their fig. 5. NT2015 is taken for a friction coefficient of 0.02 shown in their fig. 9. Shown by the red dashed line and circles are linear best fits for the last 700 Myrs, during which all vary in $Q_{c}$ by less than 3 TW/Gyrs. Blue, green, and black dashed lines show an exponential fit to the corresponding published model result. (For interpretation of the references to colour in this figure legend, the reader is referred to the web version of this article.)

(Jaupart et al., 2015) means that any dependence on the initial conditions should be lost long before the time when the inner core forms. We also attempt to mitigate any effect of initial conditions by first running each of our models backwards in time without a stable layer. Initial conditions for this backwards model are provided by present day observations, which are much better constrained than the conditions before inner core formation. Models are then run forwards in time, starting from the final state of the backwards model but with the initial core temperature adjusted to ensure the correct ICB radius at the present day. We find that the required adjustment to the initial temperature is very small, typically less than $20 \mathrm{~K}$, and so we do not expect any significant dependence of our results on the initial core temperature.

\section{Results}

We first consider an example model to demonstrate the effect of a stable layer on the thermal evolution of the core. The example has no entrainment, core parameters corresponding to $\Delta \rho=800 \mathrm{~kg} \mathrm{~m}^{-3}$ and $Q_{\mathrm{c}}$ defined by $A=10$ TW and $B=8$ TW Gyrs ${ }^{-1}$. Fig. 7 shows two models with this setup that are identical except that one includes the development of a thermally stable layer while the other does not. In this case the stable layer forms around 300 Myrs ago and grows to $400 \mathrm{~km}$ thick by the present day. The inner core forms around $700 \mathrm{Ma}$ in both models and grows to a present radius of $1231 \mathrm{~km}$ and $1221 \mathrm{~km}$ in the cases with and without a stable layer, a difference of only $10 \mathrm{~km}$ (Fig. 7a). The adiabatic region cools faster when a stable layer is present because of the slight increase in adiabatic heat flow with depth and the decrease in $\widetilde{Q}_{\mathrm{g}}$ due to the reduced volume. These effects produce a slightly larger present-day inner core. The energy terms are also similar (Fig. 7b), with changes in $Q_{\mathrm{L}}$ and $Q_{\mathrm{g}}$ of $0.71 \mathrm{TW}(+21 \%)$ and $-0.13 \mathrm{TW}(-6 \%)$ between cases with and without a layer. Although faster cooling in the stable layer case acts to increase $Q_{g}$, the reduced volume in which the light elements are distributed leads to an overall reduction in $Q_{g}$.

The associated entropy sources are shown in Fig. 7c. $E_{\mathrm{g}}=Q_{\mathrm{g}} / T_{\mathrm{c}}$ follows $Q_{g}$ and is reduced relative to the case with no stable layer. Although $Q_{\mathrm{L}}$ is increased in the presence of a layer, due to faster cooling, $E_{\mathrm{L}}$ is reduced due to the increased value for $T_{\mathrm{c}}$ in the efficiency factor $\left(T_{\mathrm{i}}\right.$ $\left.-T_{\mathrm{c}}\right) /\left(T_{\mathrm{i}} T_{\mathrm{c}}\right)$. Since no chemical effects are considered within the stable layer, the entropy due to barodiffusion, $E_{\alpha}$, is negligible in both cases as found in previous work (Gubbins et al., 2004; Davies, 2015). The largest contribution to $E_{\mathrm{k}}$ comes from the $\mathrm{CMB}$ region since the magnitude of the adiabatic gradient increases with radius and temperature decreases with radius. The presence of a stable layer therefore acts to reduce $E_{\mathrm{k}}$, by around $9 \%$ in this example. The Ohmic dissipation $E_{\mathrm{J}}$ is reduced in the presence of a stable layer because the decreases in $E_{\mathrm{L}}$ and $E_{\mathrm{g}}$ outweigh the decrease in $E_{\mathrm{k}}$.

Fig. $7 \mathrm{~d}$ shows present-day radial profiles of the potential temperature $\Theta=T_{\mathrm{s}}-T_{\mathrm{a}}$ and the Brunt-Väisälä period

$T_{B V}=\frac{2 \pi}{N}=2 \pi\left(\frac{g}{\rho} \frac{\partial \rho^{\prime}}{\partial r}\right)^{-1 / 2}=2 \pi\left(-\frac{g \alpha_{T}}{\rho} \frac{\partial \Theta}{\partial r}\right)^{-1 / 2}$

for the example case. The period depends upon the gradient of the density anomaly from the well mixed profile, $\rho^{\prime}=-\alpha_{T} \Theta$, since this is the stabilising component of the density gradient. $\Theta$ reaches $\approx 30 \mathrm{~K}$ at the top of the layer, which is much greater than the anomalies of $O\left(10^{-3}\right) \mathrm{K}$ associated with core convection (Jones, 2015). The Brunt-Väisälä period is around $24 \mathrm{~h}$ at the top of the layer, similar to predictions based on theoretical arguments (Braginsky, 1999), but weaker than values obtained for chemical stratification by Helffrich and Kaneshima (2010).

In all of our models $E_{J}$ reaches a minimum just before inner core nucleation. This places a constraint on the allowed values of $A$ for a given $B$ in order for the dynamo to have operated $\left(E_{J}>0\right)$ for the last 3.5 Gyrs. In the example shown in Fig. 7, $E_{\mathrm{J}}$ reaches a minimum of just 55 MW $\mathrm{K}^{-1}$ and so the value of $A$ cannot be reduced much further without causing $E_{\mathrm{J}}$ to fall below zero around $700 \mathrm{Ma}$. Thicker layers arise for more strongly subadiabatic conditions and hence lower $A$, but this requires larger values of $B$ in order to achieve a positive $E_{J}$ just prior to inner core nucleation.

We calculated stable layer properties for the 3 sets of core properties in Table 1. For each set we consider values of the present-day CMB heat flow $A$ in the range $6 \leq A \leq 18$ TW (Lay et al., 2009; Nimmo, 2015) and the linear heat flow gradient $B$ in the range $1 \leq B \leq 13 \mathrm{TW} \mathrm{Gyr}^{-1}$ (see Fig. 6). Fig. 8 shows the present day stable layer thickness in this parameter space for zero entrainment, $E=0$. Models in which $E_{\mathrm{J}}<0$ at any time are shown by the white space in the figure and models that produce no present-day stratification are shown by the grey colour. As expected, lower values of $A$ require larger $B$ to ensure that $E_{J}$ remains positive prior to inner core nucleation. The thickest layers correspond to the lowest values of $A$ and $B$ that ensure $E_{J}>0$. Thicker layers are allowed as $\Delta \rho$ increases, mainly because the extra gravitational power enables the dynamo to operate under more subadiabatic conditions. With $\Delta \rho=600 \mathrm{~kg} \mathrm{~m}^{-3}$ the maximum layer thickness is around $600 \mathrm{~km}$, rising to around $750 \mathrm{~km}$ at $\Delta \rho=1000 \mathrm{~kg} \mathrm{~m}^{-3}$ close to the maximum thickness obtain by Gubbins et al. (2015).

To further constrain the viable layer thickness we might consider how the recent trend in $Q_{c}$ that we have prescribed is related to the longer term trend in $Q_{\mathrm{c}}$. For the bulk of Earth's history, between roughly 1 and 4 Gyrs, the published models on Fig. 6 show an exponential decrease in $Q_{c}$ shown by the dashed lines. The histories diverge from this exponential during inner core growth since the presence of latent heat 

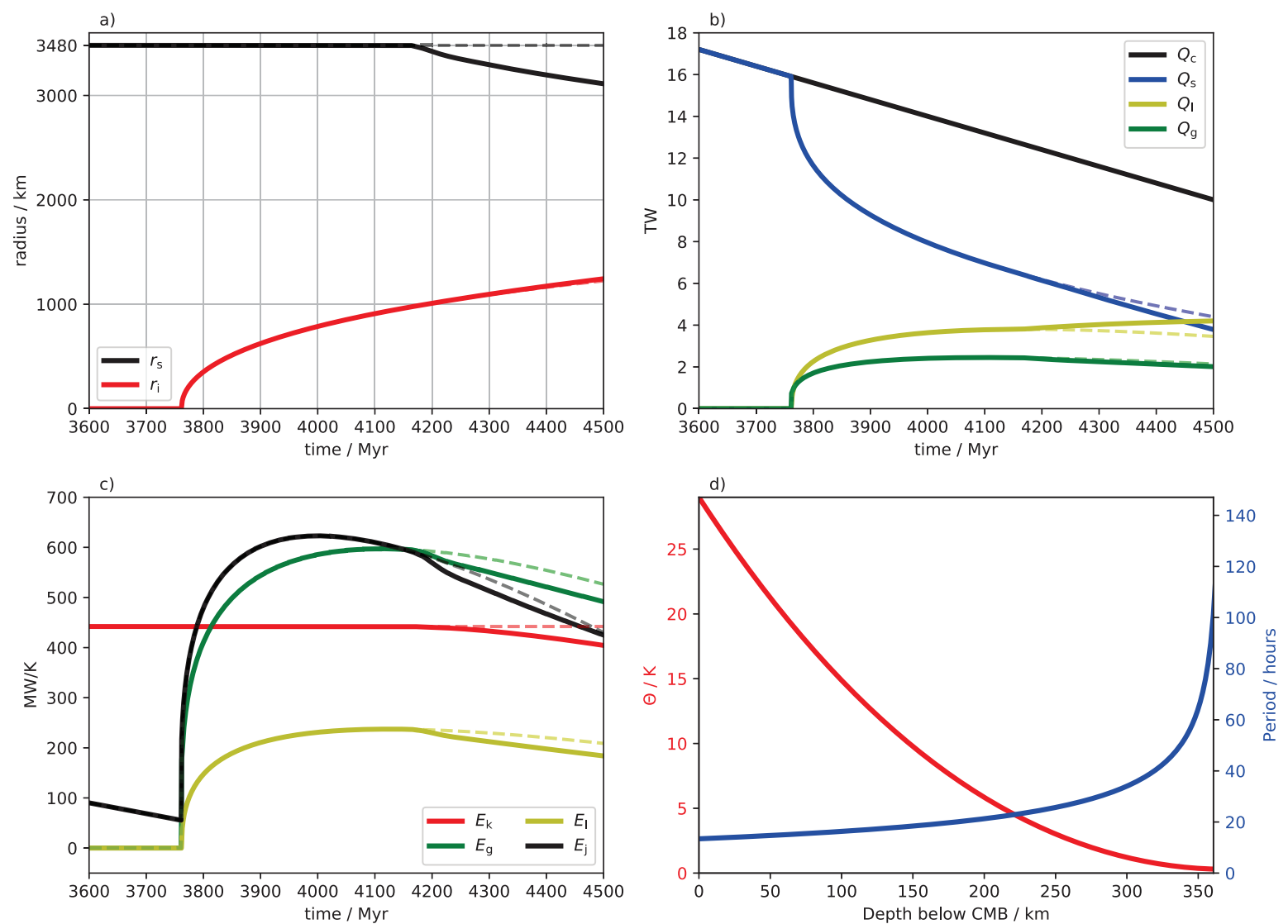

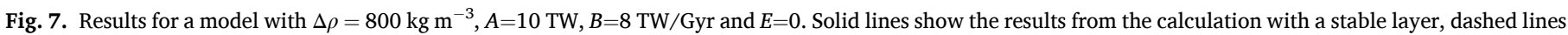

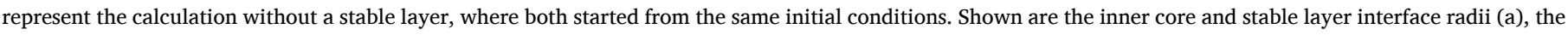
energy sources (b), the entropy sources (c) and the present day layer size and buoyancy period (d).

and gravitational energy reduces the secular cooling of the core. The temperature difference between the $\mathrm{CMB}$ and the top of the lower thermal boundary layer is relatively increased, slowing the decrease in $Q_{\mathrm{c}}$ (Driscoll and Bercovici, 2014), making the gradient of $Q_{\mathrm{c}}$ on Fig. 6 more shallow than the exponential fit. The significance of this effect on $Q_{c}$ is variable, being more noticeable in the results from Patočka et al. (2020) and Driscoll and Bercovici (2014) than from Nakagawa and Tackley (2015).

We assume that the linear time-dependence of $Q_{\mathrm{c}}$ used to obtain the results in Fig. 8 is part of an exponential variation of $Q_{c}$ over the last 3.5 Gyrs as suggested by the published time-series in Fig. 6 . For each value of $A$ and $B$ we extrapolate backwards in time along the corresponding exponential curve to obtain the value of $Q_{\mathrm{c}}$ at $3.5 \mathrm{Ga}$, denoted $Q_{\mathrm{c}}^{i}$. This assumes that inner core growth does not diverge the long term trend in $Q_{\mathrm{c}}$ from an exponential in the way described above, and therefore constitutes a lower bound on $Q_{\mathrm{c}}^{i}$. The black contours on Fig. 8 show values of $Q_{\mathrm{c}}^{i}=70,100$ and $200 \mathrm{TW}$. This extrapolation suggests that the majority of models in Fig. 8 correspond to CMB heat flows at $3.5 \mathrm{Ga}$ in excess of $100 \mathrm{TW}$, which is beyond the typically reported histories based upon coupled simulations. If we take $Q_{\mathrm{c}}^{i}=70 \mathrm{TW}$ as an upper limit on plausible heat flows (Fig. 6) then the corresponding maximum layer thickness is $\sim 250 \mathrm{~km}$ for $\Delta \rho=600 \mathrm{~kg} \mathrm{~m}^{-3}$, rising to around $450 \mathrm{~km}$ for $\Delta \rho=1000 \mathrm{~kg} \mathrm{~m}^{-3}$.

Increasing $E$ delays the onset of thermal stratification because downward entrainment of buoyant fluid can overcome a net stabilising CMB heat flow until $Q_{\mathrm{c}}<(1-E) Q_{\mathrm{a}}$. Fig. 9 shows that an entrainment factor of $E=0.2$ significantly reduces the stable layer thickness compared to the case with $E=0$ (Fig. 8). With $E=0.2$ the maximum layer thickness for $\Delta \rho=600 \mathrm{~kg} \mathrm{~m}^{-3}$ is around $300 \mathrm{~km}$, rising to around
$400 \mathrm{~km}$ for $\Delta \rho=1000 \mathrm{~kg} \mathrm{~m}^{-3}$. Extrapolating these results backwards in time, following an exponential time-dependence for $Q_{c}$ as above, suggests a maximum layer thickness of $\sim 250 \mathrm{~km}$ for a limit of $Q_{\mathrm{c}}^{i}=200 \mathrm{TW}$ on the CMB heat flow at $3.5 \mathrm{Ga}$. This reduces to $\sim 200 \mathrm{~km}$ for an upper limit of $Q_{\mathrm{c}}^{i}=100 \mathrm{TW}$; further, if $\Delta \rho=600 \mathrm{~kg} \mathrm{~m}^{-3}$, then this heat flow limit precludes present day stratification in paleomagnetically compatible models. Increasing $E$ to 0.5 causes complete entrainment of the layer for all values of $\Delta \rho$.

We take models that satisfy this constraint as being compatible with the published models in Fig. 6, limiting the selection to those models that give $Q_{\mathrm{c}}^{i}<70 \mathrm{TW}$, with maximum layer thicknesses for a range of $\Delta \rho$ and $E$ values shown in Table 3 . When $E=0$, the maximum layer thickness is $\sim 250-300 \mathrm{~km}$ for $\Delta \rho=600$ and $800 \mathrm{~kg} \mathrm{~m}^{-3}$, and $\sim 400 \mathrm{~km}$ for $\Delta \rho=1000 \mathrm{~kg} \mathrm{~m}^{-3}$. Increasing $E$ quickly lowers this upper limit since thicker layers are only found in regions of the parameter space that give progressively higher values for $Q_{\mathrm{c}}^{i}$. When $E=0.1$, the maximum layer thickness is just $<60 \mathrm{~km}$ for $\Delta \rho=600$ and $800 \mathrm{~kg} \mathrm{~m}^{-3}$, and $\sim 200 \mathrm{~km}$ for $\Delta \rho=1000 \mathrm{~kg} \mathrm{~m}^{-3}$. Only models with $\Delta \rho=1000 \mathrm{~kg} \mathrm{~m}^{-3}$ produce a stable layer when $E=0.2$, at a maximum of just $12 \mathrm{~km}$, and no models at $E=0.3$ produce a layer, given the constraint upon $Q_{\mathrm{c}}^{i}$.

Fig. 10 shows the peak Brunt-Väisälä period for all models. The maximum thermal anomaly always occurs at the present-day directly below the CMB (e.g. Fig. 7) and so the values do not depend on $B$ or $E$. Results for $\Delta \rho=600 \mathrm{~kg} \mathrm{~m}^{-3}$ and $\Delta \rho=1000 \mathrm{~kg} \mathrm{~m}^{-3}$ are similar because $k$, and hence the CMB thermal gradient, are almost the same in both cases. Values range from $8-25 \mathrm{~h}$, which is still not low enough to match the highest estimate of $3.43 \mathrm{~h}$ from (Helffrich and Kaneshima, 2010). However, the values are compatible with other estimates based on periodic variations of the magnetic field (Buffett et al., 2016). 

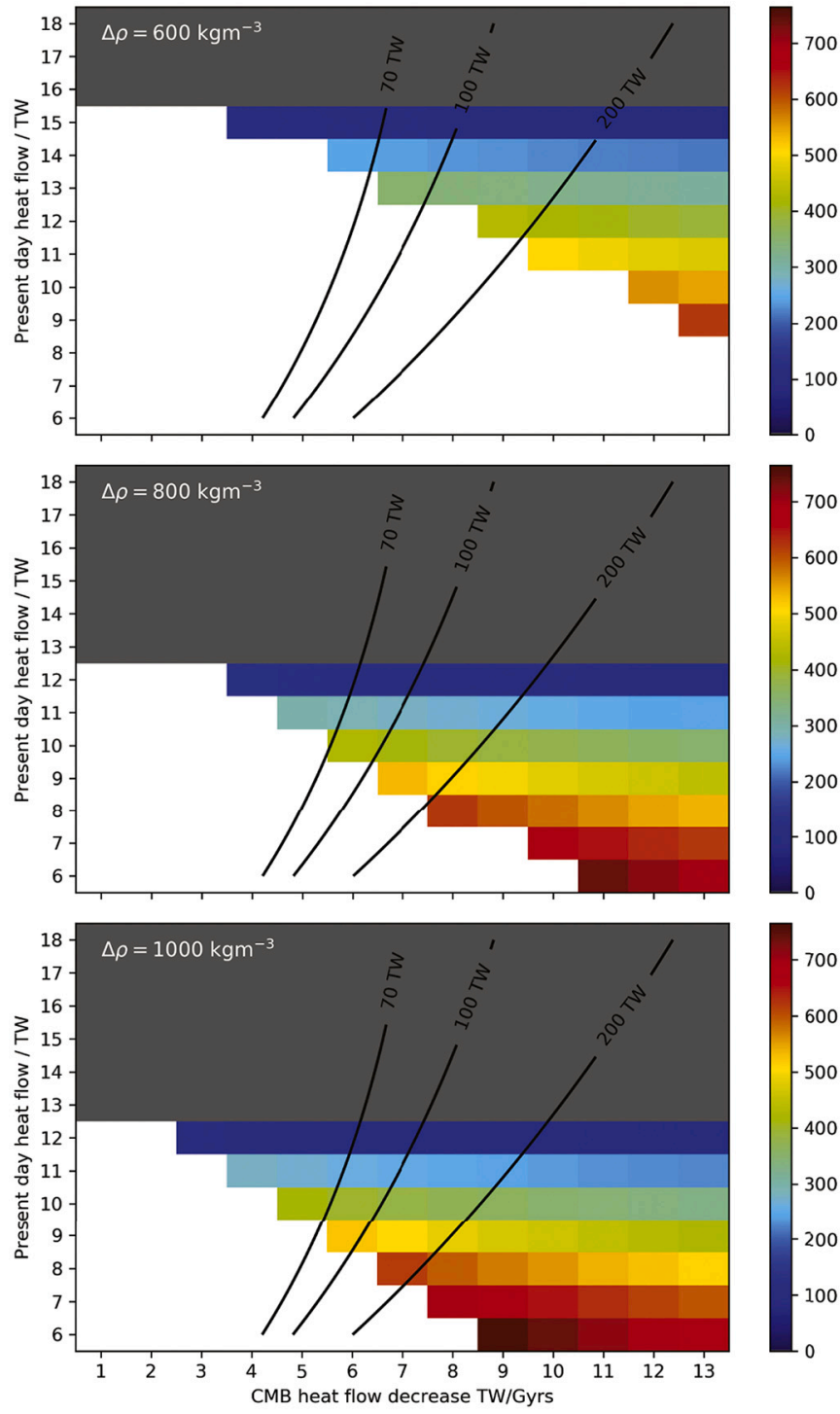

Fig. 8. Present day layer thickness for models with $\Delta \rho=600 \mathrm{~kg} \mathrm{~m}^{-3}$ (top) $\Delta \rho$ $=800 \mathrm{~kg} \mathrm{~m}^{-3}$ (middle) and $\Delta \rho=1000 \mathrm{~kg} \mathrm{~m}^{-3}$ (bottom) with $E=0$. Models in which $E_{\mathrm{J}}<0$ are ignored as shown by the white space. Grey indicates that no stable layer forms. Black contours indicate the value for $Q_{c}$ at $t=500 \mathrm{Myr}$ assuming that the present day rate of change in $Q_{c}$ were due to an exponential decay in $Q_{c}$ over the last 3.5 Gyrs (see text for details).

\section{Discussion and conclusions}

The main uncertainties in our calculations stem from the difficulty in determining core composition and $\mathrm{CMB}$ heat flow. We have considered 3 Fe-Si-O core compositions that demonstrate the effect of varying the ICB density jump within bounds constrained by current seismic observations. Composition affects the melting temperature, transport properties of the alloy such as thermal conductivity, and the gravitational energy liberated on freezing; these combined effects produce a $\sim 150 \mathrm{~km}$ change in the thickness of thermally stable layers. Other candidate elements include carbon (Badro et al., 2014) and hydrogen (Umemoto and Hirose, 2020). Recent work suggests that carbon partitions into liquid iron on freezing at ICB conditions (Li et al., 2019) and has a comparable effect to oxygen on ICB temperature and gravitational energy release, though its effect on transport properties has not been calculated. Umemoto and Hirose (2020) suggest that hydrogen becomes relevant if the ICB temperature is in the range $4800-5400 \mathrm{~K}$, which is low compared to the range 5300 - $5900 \mathrm{~K}$ considered here. Naively we might expect the
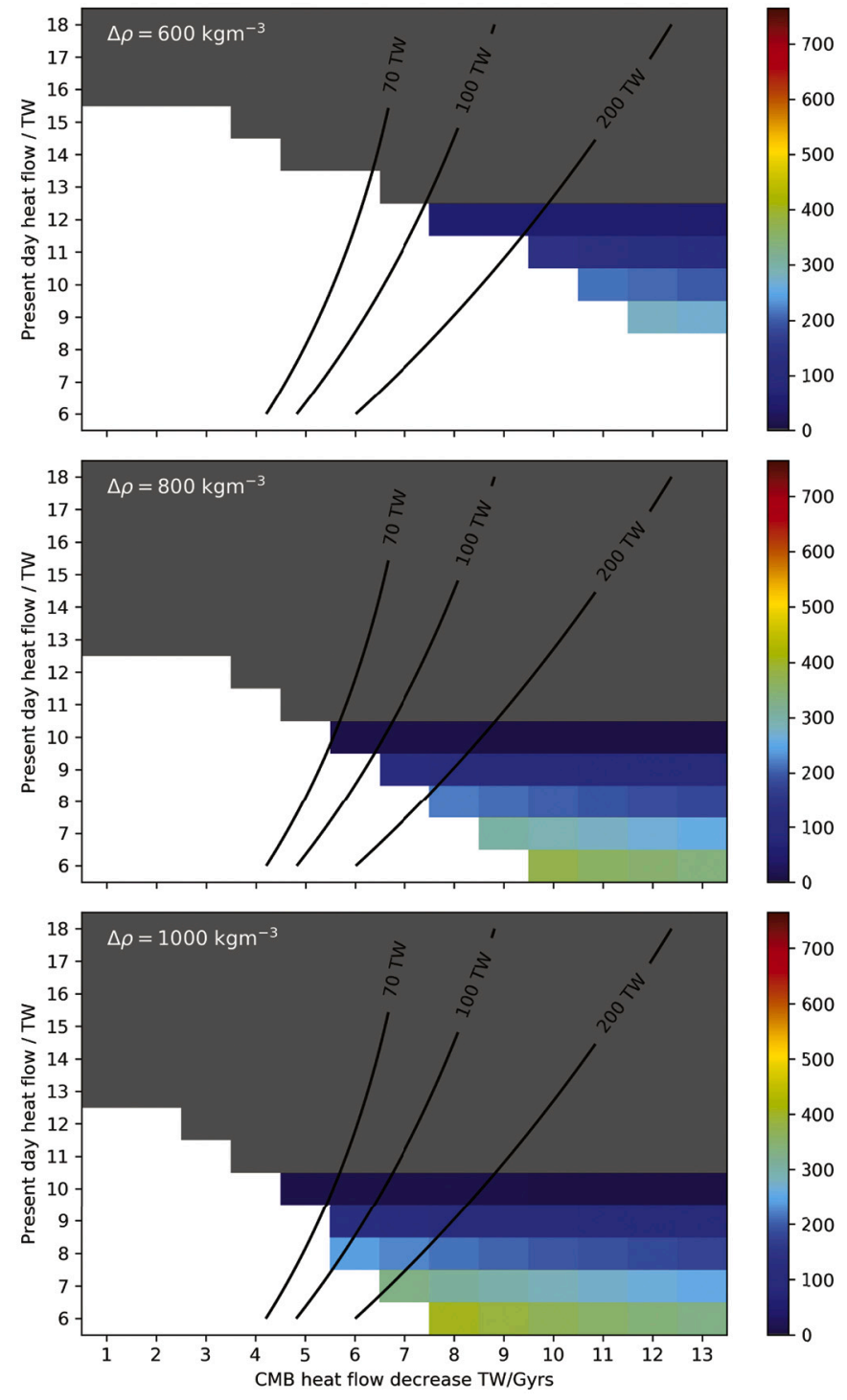

Fig. 9. Same as Fig. 8 but for $E=0.2$.

Table 3

Models producing the thickest layers at present with the requirement of $Q_{\mathrm{c}}^{i}(t=4$ $\mathrm{Ga})<70 \mathrm{TW}$.

\begin{tabular}{|c|c|c|c|c|c|c|}
\hline \multirow[b]{2}{*}{ Units } & \multirow{2}{*}{$\frac{\Delta \rho}{\mathrm{kg} \mathrm{m}^{-3}}$} & \multirow{2}{*}{$\frac{A}{\mathrm{TW}}$} & \multirow{2}{*}{$\frac{m}{\text { TW Gyr }^{-}}$} & \multirow{2}{*}{$\frac{E}{-}$} & \multirow{2}{*}{$\frac{\text { Layer size }}{\mathrm{km}}$} & \multirow{2}{*}{$\frac{Q_{\mathrm{c}}^{i}(t=3.5 \mathrm{Ga})}{\mathrm{TW}}$} \\
\hline & & & & & & \\
\hline & 600 & 14 & 6 & 0 & 246 & 63 \\
\hline & 600 & 14 & 5 & 0.1 & 28 & 49 \\
\hline & 600 & - & - & 0.2 & 0 & - \\
\hline & 600 & - & - & 0.3 & 0 & - \\
\hline & 800 & 11 & 5 & 0 & 297 & 54 \\
\hline & 800 & 11 & 5 & 0.1 & 55 & 54 \\
\hline & 800 & - & - & 0.2 & 0 & - \\
\hline & 800 & - & - & 0.3 & 0 & - \\
\hline & 1000 & 10 & 5 & 0 & 414 & 58 \\
\hline & 1000 & 10 & 5 & 0.1 & 192 & 58 \\
\hline & 1000 & 10 & 5 & 0.2 & 12 & 58 \\
\hline & 1000 & - & - & 0.3 & 0 & - \\
\hline
\end{tabular}

temperature drop from $5300 \mathrm{~K}$ to $4800 \mathrm{~K}$ to produce a similar $O(100) \mathrm{km}$ change in stable layer thickness to that found for our calculations at $5900 \mathrm{~K}$ and $5300 \mathrm{~K}$; however, this assumes that partitioning of $\mathrm{H}$ and its effect on thermal conductivity are similar to that of $\mathrm{O}$, for which there is as yet no evidence. Furthermore, Li et al. (2020) suggest from 


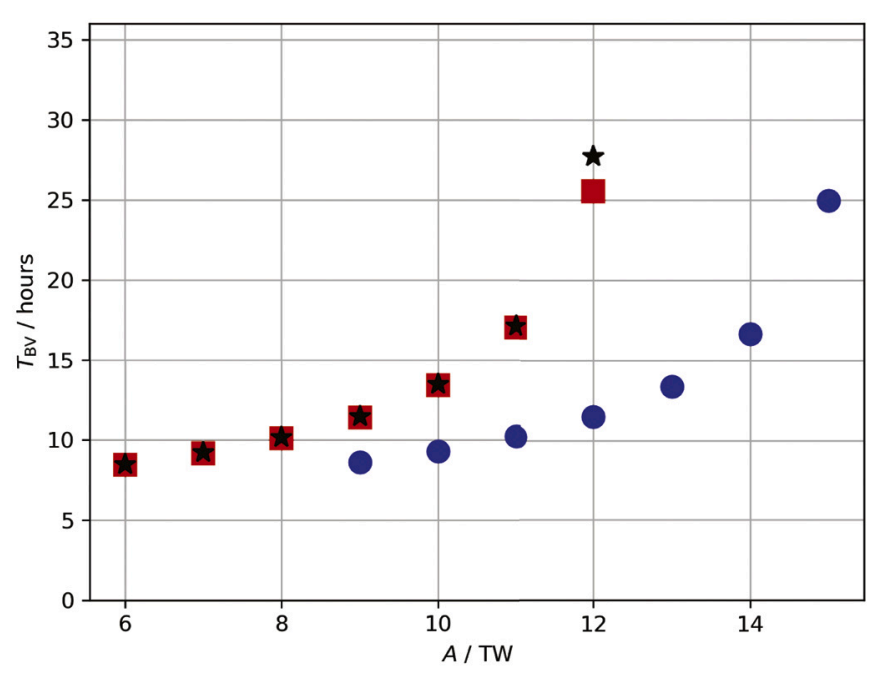

Fig. 10. Peak buoyancy period in hours for all models. No significant variation is found with $B$ or $E$ and so only models with $B=13 \mathrm{TW} / \mathrm{Gyr}$ and $E=0$ are shown. Symbols correspond to core properties $\Delta \rho=600 \mathrm{~kg} \mathrm{~m}^{-3}$ (blue circles), $\Delta \rho=800 \mathrm{~kg} \mathrm{~m}^{-3}$ (red squares) and $\Delta \rho=1000 \mathrm{~kg} \mathrm{~m}^{-3}$ (black stars). (For interpretation of the references to colour in this figure legend, the reader is referred to the web version of this article.)

partitioning calculations that the hydrogen concentrations considered by Umemoto and Hirose (2020) are too large to be compatible with the estimated present-day mantle water content. We therefore conclude that our calculations provide plausible uncertainties on the compositiondependence of stable layer thickness given the presently available information.

Much recent work has focused on the melting curve and thermal conductivity of iron and iron alloys at core conditions. Sinmyo et al. (2019) found that melting of pure iron up to $290 \mathrm{GPa}$ generally occurs at lower temperatures than the previous results (Alfè et al., 2002c; Anzellini et al., 2013) that have been used in this study. However, of greater importance for core energetics is the gradient of the pure iron melting curve, $\mathrm{d} T_{\mathrm{m}} / \mathrm{d} P$, which appears to be relatively consistent between the Sinmyo et al. (2019) and Anzellini et al. (2013) studies given uncertainties in the extrapolation to ICB pressure of $330 \mathrm{GPa}$ (see Sinmyo et al., 2019, Fig. 6). Extrapolating the Sinmyo et al. (2019) results using the Simon equation does suggest a higher $\mathrm{d} T_{\mathrm{m}} / \mathrm{d} P$ than found by Anzellini et al. (2013), which implies more inner core freezes per unit time, thus generating more latent heat and gravitational power for the dynamo. The faster growing inner core would require the inner core be younger, giving a reduced period of time when latent heat and gravitational energy are available to compliment the secular cooling in powering the dynamo. With the entropy sources in our model, thermal stratification can only form post inner core nucleation. Therefore, steeper melting curves will generally result in thinner stable layers as the layers have less time in which to form.

$\mathrm{Ab}$ initio calculations of thermal conductivity at core conditions suggest values around $100 \mathrm{~W} \mathrm{~m}^{-1} \mathrm{~K}^{-1}$ (Pozzo et al., 2013; de Koker et al., 2012; Gomi et al., 2013), though some extrapolations from lower $P-T$ find lower values of $k \approx 20-40 \mathrm{~W} \mathrm{~m}^{-1} \mathrm{~K}^{-1}$ (Konôpková et al., 2016). Lower values of $k$ reduce the thickness of thermally stable layers by reducing the heat lost down the adiabat. Since our aim is to obtain reasonable upper bounds on the layer thickness, we have focused on high $k$. With a lower $k$, lower values for the adiabatic heat flow allow lower values for $Q_{\mathrm{c}}$ whilst ensuring $E_{\mathrm{J}}>0$. Older inner cores are therefore permitted, allowing more time for thermal stratification to grow (see Labrosse et al. (1997) results in Section 2.3). Estimates for the present day $Q_{c}$ are in the range 5-17 TW (Lay et al., 2009; Nimmo, 2015), which are still above $Q_{\mathrm{a}}$ when using the data of Konôpková et al. (2016) and so would not produce thermal stratification.
CMB heat flow determinations were discussed extensively in section 3. Though the time-dependence of $Q_{c}$ is clearly not resolved by available data, we can make some reasonably firm statements. First, linear fits to recent changes in $Q_{c}$ from recent coupled core-mantle evolution models that employ high thermal conductivity (Fig. 6) produce stable layers of $O(100) \mathrm{km}$ thickness or less. Second, the thickest layers from our entire parameter search are around $750 \mathrm{~km}$, which is essentially the value obtained by considering only the present-day core (Gubbins et al., 2015). However, our results show that such thick layers cannot possibly result from an exponential time-dependence of $Q_{c}$ since this would correspond to heat flows exceeding 300 TW around $3.5 \mathrm{Ga}$, which are not predicted by any published model.

Our model of stable layer dynamics involves a simple parameterisation of entrainment by the underlying convection and also ignores double diffusive effects that may arise from thermally stable and chemically unstable conditions at the top of the core. This configuration is well known to be unstable to 'salt finger' convection (Turner, 1973; Garaud, 2018; Monville et al., 2019), promoting thin chemically enriched plumes that can rise, even though the total density stratification may be stable. Invoking additional mechanisms of entrainment would only act to reduce the thickness of a stable layer and so the results we have obtained in their absence should provide an upper bound on a thermally stable layer in Earth's core. Further investigation of these effects in 3D dynamical simulations will hopefully enable a refinement of the results we have obtained. Such simulations could also address our assumption that all gravitational energy is released in the adiabatic region of the core, though we do not expect this to bear strongly on our conclusions since the stable layer thickness remains relatively thin.

The growth of the inner core indirectly impacts the stable layer evolution in 2 ways. Firstly, it slows the cooling rate of the core through additional latent heat and liberation of gravitational energy, slowing the layer growth relative to if there was no inner core; this effect is captured in our model. Secondly, the enrichment of the convecting fluid with light elements increases its relative buoyancy and so can promote mixing of the stable layer, this would also lower its growth rate. The importance of the chemical enrichment in the convecting region on the growth rate of the stable layer depends upon the separate chemical evolution of the two regions. We believe this effect could be captured in our model and potentially related to our $E>0$ models, although this depends upon currently uncertain processes as we will discuss.

As an end member solution, one could suppose, as in Labrosse et al. (1997), that fluid incorporated into the layer as it grows does not change in composition any further and is 'frozen' in. The resulting light element profile would increase with depth in the layer and be continuous with the well mixed region at $r_{\mathrm{s}}$. The gradient of this profile throughout the layer depends on the chemical change in the well mixed region, $\mathrm{d} c l / \mathrm{d} t$, relative to the growth rate of the layer, $\mathrm{d} r_{\mathrm{s}} / \mathrm{d} t$, and at the interface will equal the ratio $\mathrm{d} c l / \mathrm{d} t \times\left(\mathrm{d} r_{\mathrm{s}} / \mathrm{d} t\right)^{-1}$ at that time. The temperature gradient required to balance this chemical gradient for neutral stability satisfies $\alpha_{T}\left(\mathrm{~d} T / \mathrm{d} r-\mathrm{d} T_{\mathrm{a}} / \mathrm{d} r\right)=\alpha_{c} \mathrm{~d} c c^{l} / \mathrm{d} r$ and taking present day values from the model presented in Fig. 7 gives a required sub-adiabatic gradient of $\mathrm{d} T$ / $\mathrm{d} r-\mathrm{d} T_{\mathrm{a}} / \mathrm{d} r=7 \times 10^{-4} \mathrm{~K} \mathrm{~m}^{-1}$. The opposite end member solution would be that the entire layer is unstable to double diffusive convection, which in this regime would produce salt-finger convection (Turner, 1973). In this scenario, despite the thermally stable profile, thin chemically enriched plumes would continuously act to mix any destabilising light element profile back towards a homogeneous configuration. The salt finger convection would ensure $\mathrm{d} c / \mathrm{d} r=0$, therefore requiring the thermal gradient in the layer to simply equal the adiabatic temperature gradient for neutral stability.

In the first, 'frozen light element', end member solution, we might represent the impact of the chemically enriched convection with the parameterisation of entrainment we have used in Eq. 22. Using the above thermal gradient of $7 \times 10^{-4} \mathrm{~K} \mathrm{~m}^{-1}$ as the representative thermal gradient resulting from such entrainment and solving for $E$ with Eq. 22 gives $E \approx 0.9$. This value is much larger than the example solutions in 
this paper and would therefore easily inhibit the growth of any thermal layer; however, it is an extreme upper bound on the influence of light element release at the ICB since it ignores any rearrangement of light element within the layer. Furthermore, this value was taken using values at the present day, i.e. when $\mathrm{d} r_{\mathrm{s}} / \mathrm{d} t$ is smaller and $\mathrm{d} c / \mathrm{d} t$ is at a maximum, giving the largest required sub-adiabatic thermal gradient and hence the largest corresponding $E$, instead of capturing any time dependence. In the second end member solution with salt finger convection acting across the entire layer, $E=0$ assuming negligible entrainment from thermal convection as well. Salt finger convection has been shown to effectively mix away light element variations in a thermal layer in Mercury's core (Manglik et al., 2010) and the same may be true for Earth, although we do not currently know this. How widespread salt finger convection could be and its efficiency at mixing or disrupting the layer are all important unanswered questions for further research. Regardless, the effect of a growing inner core would be to slow down the growth of a thermal stable layer and so would not lead to an increase in any of the upper bounds on present day layer size presented in this study.

The main result from this work is that thermally stable layers in Earth's core driven by exponentially decaying CMB heat flows are no thicker than $250-400 \mathrm{~km}$ and have maximum present-day BruntVäisälä periods, $T_{B V}=8-24 \mathrm{hrs}$. If the underlying convective region is able to significantly entrain fluid at the base of the layer, the upper bound on layer size quickly decreases to 0 by $E=0.3$. Some seismic studies that find low velocities in the upper core have obtained layer thicknesses ranging from $50-100 \mathrm{~km}$ (Lay and Young, 1990; Garnero et al., 1993). If such layers had a thermal origin they would require only moderate changes in CMB heat flow and are compatible with all core compositions considered here. More recent studies find thicker layers of up to $400 \mathrm{~km}$ (Kaneshima, 2017), which would require a present day CMB heat flow of 10-12 TW. However, producing such a thick layer while maintaining the dynamo requires a steeply dropping CMB heat flow in recent times, even when ignoring entrainment; assuming that this recent trend is part of a long-term exponential decay yields values of $Q_{\mathrm{c}}$ at $3.5 \mathrm{Ga}$ that are higher than in any recent mantle evolution model. Our results therefore suggest that such thick layers are at the upper limit and possibly exceed what can be produced by thermal stratification, at least based on current understanding of core-mantle structure and evolution.

Comparing our modelling results to seismic observations is challenging because it is likely that the small thermal anomalies, $\mathscr{O}(10) \mathrm{K}$, would not be seismically detectable and the Brunt-Väisälä period is hard to constrain from seismic data since it depends on the radial density gradient, which is not directly observed. Helffrich and Kaneshima (2010) matched their SmKS data to predictions from a thermodynamic model of the Fe-S-O system and found $T_{B V}=1.63-3.43$ hours, lower than predictions from our model. This is perhaps unsurprising since light elements are thought to have a larger effect on bulk modulus than temperature (Komabayashi, 2014). However, it does indicate that values of $T_{B V}$ are crucial to distinguishing between thermal and chemical origins of the stable layer.

Periodic variations of the geomagnetic field combined with length of day constraints have been used to advocate layers of around $130 \mathrm{~km}$ (Buffett et al., 2016) with a Brunt-Väisälä period of around $19 \mathrm{~h}$ at the CMB. From Fig. 8 the model with $\Delta \rho=800 \mathrm{~kg} \mathrm{~m}^{-3}, A=12$ TW and $B=4$ TW $\mathrm{Gyr}^{-1}$ closely matches these results. Other geomagnetic constraints based on requiring advection near the top of the core to explain some key features of the secular variation also suggest layers of $\mathscr{O}(100) \mathrm{km}$ (Gubbins, 2007). Again, these constraints can be satisfied by a large class of core models based on thermal stratification.

The key to distinguishing between thermal and compositional origins of a stable layer at the top of the core lies in improved observational determinations of the layer thickness and stratification strength. Theoretical models that attempt to explain the layer by barodiffusion of light elements down the pressure gradient (Fearn and Loper, 1981; Gubbins and Davies, 2013) or partitioning of $\mathrm{FeO}$ into the core from the mantle (Buffett and Seagle, 2010; Davies et al., 2018, 2020) predict layers of $\mathscr{O}(100) \mathrm{km}$, the thickness being limited by the small chemical diffusion coefficients. Chemical layers arising from turbulent mixing during core formation may produce $300 \mathrm{~km}$-thick layers (Landeau et al., 2016), similar to the thermal layers studied here, however it is currently unclear whether such thick chemical layers would survive late giant impacts (Jacobson et al., 2017). Chemical models also predict that $T_{B V}$ is much lower than values of $8-24$ hours obtained here: Buffett and Seagle (2011) obtained $T_{B V} \approx 0.5$ hours, while Gubbins and Davies (2013) found $T_{B V} \approx 1$ hour for their chemical layers. Seismic observations can also be used to look for regional variations in the strength and structure of core stratification, which may point to the influence of lateral heat flow variations at the CMB (Mound et al., 2019).

\section{Declaration of Competing Interest}

The authors declare that they have no known compe5ng financial interests or personal rela5onships that could have appeared to influence the work reported in this paper.

\section{Acknowledgements}

We are grateful to the two anonymous reviews who provided useful insight that helped significantly improve this manuscript. SG acknowledges funding from the Natural Environment Research Council SPHERES Doctoral Training Program. CD acknowledges a Natural Environment Research Council personal fellowship, reference NE/ L011328/1. Figures were made using Matplotlib (Hunter, 2007).

\section{References}

Alexandrakis, C., Eaton, D., 2010. Precise seismic-wave velocity atop Earth's core: no evidence for outer-core stratification. Phys. Earth Planet. Int. 180, 59-65.

Alfè, D., Price, G., Gillan, M., 2001. Thermodynamics of hexagonal close packed iron under Earth's core conditions. Phys. Rev. B 64, 045123.

Alfe, D., Gillan, M., Price, G., 2002a. Composition and temperature of the Earth's core constrained by combining ab initio calculations and seismic data. Earth Planet. Sci. Lett. 195, 91-98.

Alfè, D., Gillan, M., Price, G., 2002b. Ab initio chemical potentials of solid and liquid solutions and the chemistry of the Earth's core. J. Chem. Phys. 116, 7127-7136.

Alfè, D., Price, G., Gillan, M., 2002c. Iron under Earth's core conditions: liquid-state thermodynamics and high-pressure melting curve from $a b$ initio calculations. Phys. Rev. B 65, 165118.

Anzellini, S., Dewaele, A., Mezouar, M., Loubeyre, P., Morard, G., 2013. Melting of iron at Earth's inner core boundary based on fast x-ray diffraction. Science 340, 464-466.

Aubert, J., Finlay, C., Fournier, A., 2013. Bottom-up control of geomagnetic secular variation by the Earth's inner core. Nature 502, 219-223.

Badro, J., Côté, A., Brodholt, J., 2014. A seismologically consistent compositional model of Earth's core. Proc. Natl. Acad. Sci. 111, 7542-7545.

Badro, J., Siebert, J., Nimmo, F., 2016. An early geodynamo driven by exsolution of mantle components from Earth's core. Nature 536, 326.

Badro, J., Aubert, J., Hirose, K., Nomura, R., Blanchard, I., Borensztajn, S., Siebert, J., 2018. Magnesium partitioning between Earth's mantle and core and its potential to drive an early exsolution geodynamo. Geophys. Res. Lett. 45, 13-24.

Bouffard, M., Choblet, G., Labrosse, S., Wicht, J., 2019. Chemical convection and stratification in the Earth's outer core. Front. Earth Sci. 7, 99.

Bouffard, M., Landeau, M., Goument, A., 2020. Convective erosion of a primordial stratification atop earth's core. Geophys. Res. Lett. 47 e2020GL087109.

Braginsky, S., 1963. Structure of the F layer and reasons for convection in the Earth's core. Sov. Phys. Dokl. 149, 8-10.

Braginsky, S., 1999. Dynamics of the stably stratified ocean at the top of the core. Phys. Earth Planet. Int. 111, 21-34.

Braginsky, S., Roberts, P., 1995. Equations governing convection in Earth's core and the geodynamo. Geophys. Astrophys. Fluid Dyn. 79, 1-97.

Brodholt, J., Badro, J., 2017. Composition of the low seismic velocity E' layer at the top of Earth's core. Geophys. Res. Lett. 44, 2017 GL074261.

Buffett, B., Seagle, C., 2010. Stratification of the top of the core due to chemical interactions with the mantle. J. Geophys. Res. 115, B04407.

Buffett, B.A., Seagle, C.T., 2011. Correction to "stratification of the top of the core due to chemical interactions with the mantle". J. Geophys. Res. 116.

Buffett, B., Knezek, N., Holme, R., 2016. Evidence for MAC waves at the top of Earth's core and implications for variations in length of day. Geophys. J. Int. 204, 1789-2000. https://doi.org/10.1093/gii/ggv552.

Christensen, U., 2006. A deep dynamo generating Mercury's magnetic field. Nature 444, 1056-1058. 
Conrad, C.P., Hager, B.H., 1999. The thermal evolution of an Earth with strong subduction zones. Geophys. Res. Lett. 26, 3041-3044.

Crank, J., 1979. The Mathematics of Diffusion. Oxford University Press.

Davies, C., 2015. Cooling history of Earth's core with high thermal conductivity. Phys. Earth Planet. Int. 247, 65-79.

Davies, C., Gubbins, D., 2011. A buoyancy profile for the Earth's core. Geophys. J. Int. $187,549-563$.

Davies, C.J., Mound, J.E., 2019. Mantle-induced temperature anomalies do not reach the inner core boundary. Geophys. J. Int. 219, S21-S32.

Davies, C., Pommier, A., 2018. Iron snow in the martian core? Earth planet. Sci. Lett. 481, 189-200.

Davies, C., Pozzo, M., Gubbins, D., Alfè, D., 2015. Constraints from material properties on the dynamics and evolution of Earth's core. Nat. Geosci. 8, 678-687.

Davies, C., Pozzo, M., Gubbins, D., Alfè, D., 2018. Partitioning of oxygen between ferropericlase and Earth's liquid core. Geophys. Res. Lett. 45, 6042-6050.

Davies, C.J., Pozzo, M., Gubbins, D., Alfè, D., 2020. Transfer of oxygen to Earth's core from a long-lived magma ocean. Earth Planet. Sci. Lett. 538, 116208.

de Koker, N., Steinle-Neumann, G., Vojtech, V., 2012. Electrical resistivity and thermal conductivity of liquid Fe alloys at high $\mathrm{P}$ and $\mathrm{T}$ and heat flux in Earth's core. Proc. Natl. Acad. Sci. 109, 4070-4073.

Driscoll, P., Bercovici, D., 2014. On the thermal and magnetic histories of Earth and Venus: influences of melting, radioactivity, and conductivity. Phys. Earth Planet. Int 236, 36-51.

Du, Z., Boujibar, A., Driscoll, P., Fei, Y., 2019. Experimental constraints on an MgO exsolution-driven geodynamo. Geophys. Res. Lett. 46, 7379-7385.

Dumberry, M., Rivoldini, A., 2015. Mercury's inner core size and core-crystallization regime. Icarus 248, 254-268. https://doi.org/10.1016/j.icarus.2014.10.038.

Dziewonski, A., Anderson, D., 1981. Preliminary reference earth model. Phys. Earth Planet. Int. 25, 297-356.

Fearn, D., Loper, D., 1981. Compositional convection and stratification of Earth's core. Nature 289, 393-394.

Garaud, P., 2018. Double-diffusive convection at low Prandtl number. Annu. Rev. Fluid Mech. 50, 275-298.

Garnero, E.J., Helmberger, D.V., Grand, S.P., 1993. Constraining outermost core velocity with SmKS waves. Geophys. Res. Lett. 20, 2463-2466.

Gomi, H., Ohta, K., Hirose, K., Labrosse, S., Caracas, R., Verstraete, V., Hernlund, J., 2013. The high conductivity of iron and thermal evolution of the Earth's core. Phys. Earth Planet. Int. 224, 88-103.

Gubbins, D., 2004. Time Series Analysis and Inverse Theory for Geophysicists. Cambridge University Press.

Gubbins, D., 2007. Geomagnetic constraints on stratification at the top of Earth's core. Earth Planets Space 59, 661-664.

Gubbins, D., Davies, C., 2013. The stratified layer at the core-mantle boundary caused by barodiffusion of oxygen, sulphur and silicon. Phys. Earth Planet. Int. 215, 21-28.

Gubbins, D., Roberts, P.H., 1987. Magnetohydrodynamics of Earth's core. In: Jacobs, J.A. (Ed.), Geomagnetism. Academic Press, pp. 1-187.

Gubbins, D., Thomson, C., Whaler, K., 1982. Stable regions in the Earth's liquid core. Geophys. J. R. Astr. Soc. 68, 241-251.

Gubbins, D., Alfe, D., Masters, G., Price, G., Gillan, M., 2003. Can the Earth's dynamo run on heat alone? Geophys. J. Int. 155, 609-622.

Gubbins, D., Alfè, D., Masters, G., Price, G., Gillan, M., 2004. Gross thermodynamics of two-component core convection. Geophys. J. Int. 157, 1407-1414.

Gubbins, D., Alfè, D., Davies, C., Pozzo, M., 2015. On core convection and the geodynamo: effects of high electrical and thermal conductivity. Phys. Earth Planet. Int. 247, 56-64. https://doi.org/10.1016/j.pepi.2015.04.002.

Helffrich, G., Kaneshima, S., 2010. Outer-core compositional stratification from observed core wave speed profiles. Nature 468, 807-809. https://doi.org/10.1038/ nature09636.

Helffrich, G., Kaneshima, S., 2013. Causes and consequences of outer core stratification. Phys. Earth Planet. Int. 223, 2-7.

Hirose, K., Morard, G., Sinmyo, R., Umemoto, K., Hernlund, J., Helffrich, G., Labrosse, S., 2017. Crystallization of silicon dioxide and compositional evolution of the Earth's core. Nature 543, 99-102.

Hunter, J.D., 2007. Matplotlib: a 2d graphics environment. Comput. Sci. Eng. 9, 90-95. https://doi.org/10.1109/MCSE.2007.55.

Irving, Jessica CE, Cottaar, Sanne, Lekić, Vedran, 2018. Seismically determined elastic parameters for Earth's outer core. Sci. Adv. 4 (Article eaar2538) https://doi.org/ 10.1126/sciadv.aar2538.

Jacobson, S., Rubie, D., Herlund, J., Morbidelli, A., Nakajima, M., 2017. Formation, stratification, and mixing of the cores of Earth and Venus. Earth Planet. Sci. Lett. 474, 375-386.

Jaupart, C., Labrosse, S., Mareschal, J.C., 2015. Temperatures, heat and energy in the mantle of the Earth. In: Schubert, G. (Ed.), Treatise on Geophysics, vol. 7. Elsevier, Amsterdam, pp. 223-270.

Jones, C., 2015. Thermal and compositional convection in the outer core. In: Schubert, G. (Ed.), Treatise on Geophysics, Vol. 8, Ch. 5. Elsevier, pp. 116-159.

Kaneshima, S., 2017. Array analysis of SmKS waves and stratification of Earth's outermost core. Phys. Earth Planet. Int. 223, 2-7.

Knibbe, J., van Westrenen, W., 2018. The thermal evolution of Mercury's Fe-Si core. Earth Planet. Sci. Lett. 482, 147-159.

Komabayashi, T., 2014. Thermodynamics of melting relations in the system Fe-FeO at high pressure: implications for oxygen in the Earth's core. J. Geophys. Res. 119, 4164-4177. https://doi.org/10.1002/2014JB010980.
Konôpková, Z., McWilliams, R., Gómez-Pérez, N., Goncharov, A., 2016. Direct measurement of thermal conductivity in solid iron at planetary core conditions. Nature 534, 99-101.

Labrosse, S., Poirier, J.P., Le Mouël, J.L., 1997. On cooling of the Earth's core. Phys. Earth Planet. Int. 99, 1-17.

Labrosse, S., Poirier, J.P., Le Mouël, J.L., 2001. The age of the inner core. Earth Planet. Sci. Lett. 190, 111-123.

Landau, L., Lifshitz, E., 1987. Fluid Mechanics (Course of Theoretical Physics, Volume 6). Permagon Press, Second ed.

Landeau, M., Olson, P., Deguen, R., Hirsh, B.H., 2016. Core merging and stratification following giant impact. Nat. Geosci. 1, 1-5. https://doi.org/10.1038/NGEO2808.

Laneuville, M., Wieczorek, M., Breuer, D., Aubert, J., Morard, G., Rückriemen, T., 2014. A long-lived lunar dynamo powered by core crystallization. Earth Planet. Sci. Lett. 401, 251-260.

Lay, T., Young, C., 1990. The stably-stratified outermost core revisited. Geophys. Res. Lett. 71, 2001-2004.

Lay, T., Hernlund, J., Buffett, B., 2009. Core-mantle boundary heat flow. Nat. Geosci. 1, 25-32.

Levy, M., Fernando, H., 2002. Turbulent thermal convection in a rotating stratified fluid. J. Fluid Mech. 467, 19.

Li, Y., Vočadlo, L., Alfè, D., Brodholt, J., 2019. Carbon partitioning between the earth's inner and outer core. J. Geophys. Res. Solid Earth 124, 12812-12824.

Li, Y., Vočadlo, L., Sun, T., Brodholt, J.P., 2020. The Earth's core as a reservoir of water. Nat. Geosci. 1-6.

Lister, J., 1995. On penetrative convection at low Péclet number. J. Fluid Mech. 292, 229-248.

Lister, J., Buffett, B., 1998. Stratification of the outer core at the core-mantle boundary. Phys. Earth Planet. Int. 105, 5-19.

Manglik, A., Wicht, J., Christensen, U., 2010. A dynamo model with double diffusive convection for Mercury's core. Earth Planet. Sci. Lett. 209, 619-628.

Masters, G., Gubbins, D., 2003. On the resolution of density within the Earth. Phys. Earth Planet. Int. 140, 159-167.

Mittal, T., Knezek, N., Arveson, S.M., McGuire, C.P., Williams, C.D., Jones, T.D., Li, J. 2020. Precipitation of multiple light elements to power Earth's early dynamo. Earth Planet. Sci. Lett. 532, 116030.

Moffatt, H., Loper, D., 1994. The magnetostrophic rise of a buoyant parcel in the Earth's core. Geophys. J. Int. 117, 394-402.

Monville, R., Vidal, J., Cébron, D., Schaeffer, N., 2019. Rotating double-diffusive convection in stably stratified planetary cores. Geophys. J. Int. 219, S195-S218.

Mound, J., Davies, C., Rost, S., Aurnou, J., 2019. Regional stratification at the top of Earth's core due to core-mantle boundary heat flux variations. Nat. Geosci. 12, $575-580$.

Nakagawa, T., 2018. On the thermo-chemical origin of the stratified region at the top of the Earth's core. Phys. Earth Planet. Int. 276, 172-181.

Nakagawa, T., Tackley, P., 2007. Lateral variations in CMB heat flux and deep mantle seismic velocity caused by a thermal-chemical-phase boundary layer in 3D spherical convection. Earth Planet. Sci. Lett. 271, 348-358.

Nakagawa, T., Tackley, P.J., 2015. Influence of plate tectonic mode on the coupled thermochemical evolution of Earth's mantle and core. Geochem. Geophys. Geosyst. https://doi.org/10.1002/2015GC005996.

Nimmo, F., 2015. Energetics of the core. In: Schubert, G. (Ed.), Treatise on Geophysics, 2nd ednvol. 8. Elsevier, Amsterdam, pp. 27-55.

Nimmo, F., Price, G., Brodholt, J., Gubbins, D., 2004. The influence of potassium on core and geodynamo evolution. Geophys. J. Int. 156, 363-376.

O'Rourke, J.G., Stevenson, D.J., 2016. Powering Earth's dynamo with magnesium precipitation from the core. Nature 529, 387-389. https://doi.org/10.1038/ nature16495.

O'Rourke, J., Korenaga, J., Stevenson, D., 2017. Thermal evolution of Earth with magnesium precipitation in the core. Earth Planet. Sci. Lett. 458, 263-272.

Patočka, V., Srámek, O., Tosi, N., 2020. Minimum heat flow from the core and thermal evolution of the Earth. Phys. Earth Planet. Inter. 305.

Pozzo, M., Davies, C., Gubbins, D., Alfè, D., 2013. Transport properties for liquid siliconoxygen-iron mixtures at Earth's core conditions. Phys. Rev. B 87, 014110.

Rückriemen, T., Breuer, D., Spohn, T., 2015. The Fe snow regime in Ganymede's core: A deep-seated dynamo below a stable snow zone. J. Geophys. Res. 120 https://doi. org/10.1002/2014JE004781.

Sinmyo, R., Hirose, K., Ohishi, Y., 2019. Melting curve of iron to 290 gpa determined in a resistance-heated diamond-anvil cell. Earth Planet. Sci. Lett. 510, 45-52.

Stevenson, D., 1987. Limits on lateral density and velocity variations in the Earth's outer core. Geophys. J. Int. 88, 311-319.

Stevenson, D., 2001. Mars' core and magnetism. Nature 412, 214-219.

Tarduno, J., Cottrell, R., Watkeys, M., Hofmann, A., Doubrovine, P., Mamajek, E., Liu, D., Sibeck, D., Neukirch, L., Usui, Y., 2010. Geodynamo, solar wind, and magnetopause 3.4 to 3.45 billion years ago. Science $327,1238-1240$.

Turner, J., 1973. Buoyancy Effects in Fluids. Cambridge University Press.

Umemoto, K., Hirose, K., 2020. Chemical compositions of the outer core examined by first principles calculations. Earth Planet. Sci. Lett. 531, 116009.

Wang, D., 2003. Entrainment laws and a bulk mixed layer model of rotating convection derived from large-eddy simulations. Geophys. Res. Lett 30.

Williams, J.P., Nimmo, F., 2004. Thermal evolution of the Martian core: implications for an early dynamo. Geology 32, 97-100.

Xiong, Z., Tsuchiya, T., Taniuchi, T., 2018. Ab initio prediction of potassium partitioning into Earth's core. J. Geophys. Res. 123, 6451-6458. 